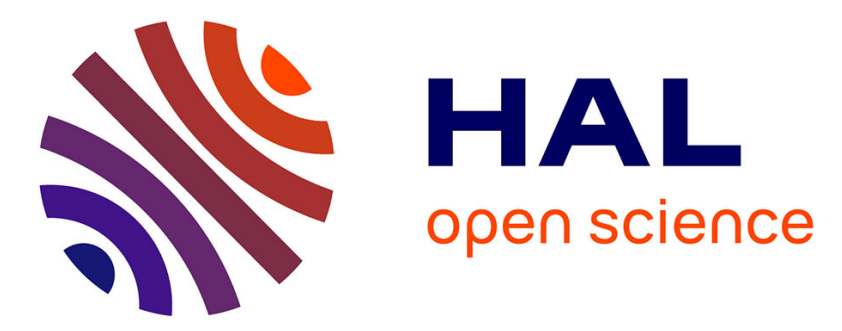

\title{
Classification of remote sensing data with morphological attributes profiles: a decade of advances
}

\author{
Deise Santana Maia, Minh-Tan Pham, Erchan Aptoula, Florent Guiotte,
} Sébastien Lefèvre

\section{- To cite this version:}

Deise Santana Maia, Minh-Tan Pham, Erchan Aptoula, Florent Guiotte, Sébastien Lefèvre. Classification of remote sensing data with morphological attributes profiles: a decade of advances. IEEE geoscience and remote sensing magazine, 2021, 9 (3), pp.43-71. 10.1109/MGRS.2021.3051859 . hal03199357

\section{HAL Id: hal-03199357 \\ https://hal.science/hal-03199357}

Submitted on 15 Apr 2021

HAL is a multi-disciplinary open access archive for the deposit and dissemination of scientific research documents, whether they are published or not. The documents may come from teaching and research institutions in France or abroad, or from public or private research centers.
L'archive ouverte pluridisciplinaire HAL, est destinée au dépôt et à la diffusion de documents scientifiques de niveau recherche, publiés ou non, émanant des établissements d'enseignement et de recherche français ou étrangers, des laboratoires publics ou privés. 


\title{
Classification of remote sensing data with morphological attributes profiles: a decade of advances
}

\author{
Deise Santana Maia, Minh-Tan Pham, Erchan Aptoula, Florent Guiotte, Sébastien Lefèvre
}

\begin{abstract}
Morphological attribute profiles (APs) are among the most prominent methods for the spatial-spectral pixel analysis of remote sensing images. Since their introduction a decade ago to tackle land-cover classification, many studies have been contributed to the state-of-the-art, focusing not only on their application to a wider range of tasks, but on their performance improvement and extension to more complex earth observation data as well. Despite the overwhelming proliferation of deep learning-based methods in the past five years, APs are far from obsolete, due mainly to their high flexibility, low computational cost, lower training data requirement, and rigorous mathematical foundation. In this survey, an entire decade of more than $\mathbf{1 0 0}$ AP related contributions to the field of remote sensing have been compiled, providing an extensive panorama of this robust and effective tool. Moreover, a collective experimental comparison of the reviewed AP variations is provided as well, not only in terms of classification performance, but for the first time in terms of their generalization capacity too.
\end{abstract}

Index Terms-Mathematical morphology, attribute profiles, multilevel image description, image classification, remote sensing.

\section{INTRODUCTION}

Classification constitutes one of the paramount tasks of remote sensing image analysis for earth observation. Its performance is critical for the success of land-use and land-cover mapping and monitoring. The rapid development of high resolution (HR) and very high resolution (VHR) image acquisition technologies, has led to increasingly more complex images and higher levels of detail. Consequently, the importance of the joint exploitation of spatially contextual information along with spectral pixel characteristics has become clear early on [1].

Morphological profiles (MPs) [2] were introduced almost two decades ago to address this exact issue. In essence, they produce multi-scale descriptions of their input, through the application of a sequence of morphological reconstruction based filters using structuring elements (SEs) of various sizes (and shapes) [2]. Their rigorous mathematical foundation, and inherent ability to capture spatial-spectral information, has led to the development of several variants [3]-[6].

However, as spatial resolutions and image sizes progressively increased, their relatively high computational cost has been drastically accentuated. Moreover, their initially celebrated capacity of capturing size and shape variations, became eventually extremely limited, when confronted with thematic classes differing in terms of alternative properties, such as contrast, homegeneity, etc. In an effort to overcome the aforementioned shortcomings, MPs have been generalized into morphological attribute profiles (APs) through the seminal work of M. Dalla Mura, J. A. Benediktsson, B. Waske and L. Bruzzone [7].

On the contrary of MPs, APs employ attribute filters (AFs) [8], a powerful class of connected morphological filters, capable of removing entire connected components w.r.t. arbitrarily defined attributes (e.g. geometric, statistical, etc), thus eliminating the size/shape limitation of MPs. Furthermore, APs can be generated efficiently through quasi-linear [9] and parallel [10] algorithms, through their input's tree-based hierarchical representation [11], thus equipping them with a high level of scalability; an invaluable property in remote sensing, where gigapixel images are becoming the norm. In fact, [12] presents an application of AP at a terapixel scale!

It is thus not surprising that since their introduction ten years ago, a great number of AP related publications have appeared (4540 at Google Scholar as of June 2020), tackling various aspects of remote sensing image analysis. Many among them have been dedicated to improving further APs through a rich variety of extensions, focusing on every single stage of their calculation.

Even though recent years have witnessed the overwhelming proliferation of deep learning [13], overshadowing performance-wise most non-deep feature extraction methods, APs continue to withstand the test of time. Besides their aforementioned invaluable properties, this is additionally due to their ability to perform even with limited amounts of training data as well as their capacity to accommodate arbitrary modalities and challenging types of images, so often encountered in the remote sensing domain.

This article $\rrbracket^{1}$ presents a survey on APs and contributes to the state of the art in the following ways:

1) We provide a comprehensive review of ten years of advances on APs, by decomposing their calculation into four stages, and grouping the reviewed studies accordingly. Evidently, this is not the first survey on APs [14], [15]. Contrary to [14] from 5 years ago, this survey is not limited with the vanilla definition of APs and their focus on hyperspectral data, and compared to [15], it additionally addresses APs on partition tree structures, threshold selection techniques and AP post-processing.

2) We present the results of an extensive series of classification experiments with multiple real datasets, intended

\footnotetext{
${ }^{1} \mathrm{~A}$ short version of this article has appeared at ICPRAI 2018
} 
to measure the performance of the various reviewed $\mathrm{AP}$ variants. However, the experiments have been conducted for the first time in terms of connectivity type as well as spectral quantization level.

3) More importantly, we present the results of pixel classification experiments intended to measure not only the performance of the various reviewed AP variants, but their generalization capacity as well. Being in the Big Data era, generalization is a core and sought after property of any content description tool, and one for which AP have been criticized for, as an image's tree representation contains both training and testing elements [16]. Mixing training/testing sets is unfortunately not uncommon with deep learning either [17]. We underline this validation malpractice, often encountered in the state of the art and propose a solution through an image's spatial subdivision and independent tree construction.

4) In order to promote reproducible research and ease generalization, we also provide the first available opensource library for APs (SAP - Simple Attribute Profiles)

In the remainder of this article, we first recall the theoretical background of APs and highlight the key stages of AP construction (Sec. II). Then we present and discuss the plethora of AP oriented developments that have taken place in the last ten years (Sec. III). Next, we present the results of our experimental study (Sec. IV) providing an extensive evaluation of some of the reviewed AP extensions, compared to the original APs. To this end, we have employed two publicly available real remote sensing image datasets and used an open source library, thus guaranteeing reproducibility. Then, we present a critical analysis of the experimental settings commonly used by AP-based classification approaches (Sec. V), underline a validation malpractice involving the mixing of training and testing data and propose an amendment. Sec. VII is devoted to concluding remarks and future research directions.

\section{PRINCIPLE OF APS}

APs are multilevel image description tools obtained by successively applying a set of morphological attribute filters (AFs) [7]. Unlike usual image filtering operators which are directly performed on pixel level, AFs work on connected component (CC) level based on the concept of image connectivity. In particular, AFs are applied on CCs with regard to a predicate based on an arbitrary statistical or geometric property thereof. Consequently, they exhibit a higher level of flexibility w.r.t. operators by reconstruction, that are severely limited with characterizing only the size and shape of their input. This advantage naturally extends to APs vs MPs as well [2], 17].

The generation of APs [7] from an input image can be summarized as a four-step process (see Fig. 1):

1) Construction of the image's hierarchical tree representation, where CCs are denoted as nodes.

${ }^{2}$ https://gitlab.inria.fr/fguiotte/sap
2) Computation of one or more relevant attributes describing the geometrical and statistical features from each tree node.

3) Filtering the tree by preserving/removing nodes according to their attribute values compared against predefined thresholds.

4) Reconstruction of the image from the filtered tree.

Step (1) can be performed using different pixel connectivity rules. For 2D images, 4 and 8-connectivity are the most common. Steps (3) and (4) can be implemented for different attributes (with different threshold values) to finally produce a set of filtered images (by stacking them) forming the APs.

More formally, according to the seminal work of [7], given a grayscale image $X: E \rightarrow \mathbb{Z}, E \subseteq \mathbb{Z}^{2}$, the calculation of APs on $X$ is achieved by applying a sequence of AFs based on a min-tree (i.e. attribute thickening operators $\left\{\phi_{k}\right\}_{k=1}^{K}$ ) and on a max-tree (i.e. attribute thinning operators $\left\{\gamma_{k}\right\}_{k=1}^{K}$ ) as follows:

$$
\begin{array}{r}
\operatorname{AP}(X)=\left\{\phi_{K}(X), \phi_{K-1}(X), \ldots, \phi_{1}(X), X,\right. \\
\left.\gamma_{1}(X), \ldots, \gamma_{K-1}(X), \gamma_{K}(X)\right\},
\end{array}
$$

where $\phi_{k}(X)$ denotes the filtered image obtained by applying the attribute thickening $\phi$ with regard to the threshold $k$. Similar explanation is made for $\gamma_{k}(X)$. As observed, the resulting $\operatorname{AP}(X)$ is a stack of $(2 K+1)$ images including the original image, $K$ filtered images from the thickening profiles and the other $K$ from the thinning profiles.

A toy example of AFs is presented in Figure 2. Given the grayscale image $X: E \rightarrow[0,1,2]$ of Figure 2(a), we first obtain the max-tree $T$ of $X$ using 4-connectivity. Then we compute the area (number of pixels) of the nodes of $T$. Subsequently, we prune the nodes of $T$ with area less than a given parameter $k$. In our case, $k$ is equal to 8 and the nodes composed of less than 8 pixels are pruned from the tree. Finally, we reconstruct the image from the pruned tree, resulting in $X^{\prime}$. Hence, $X^{\prime}$ is the area thinning of $X$ for $k=8$.

\section{RECENT ADVANCES FROM APS}

Each of the aforementioned four AP construction stages have received various forms of extensions and contributions from the scientific community. Moreover, AP based image analysis pipelines include often some form of pre-processing, usually in order to adapt multi-band input (since Eq. (1) expects single-band data) as well as post-processing steps to increase description capability.

We now revisit the recently proposed developments that have provided significant contributions to the AP framework for remote sensing image classification (Sec. III-A to Sec. III-E). Here, our study will focus on the following key concepts:

- the adaptation of APs to various modalities besides single-band images, and in particular to multi-band data (Sec. III-A);

- the construction of APs using various hierarchical image representations (Sec. III-B;

- the determination of attributes and thresholds (Sec. III-C); 


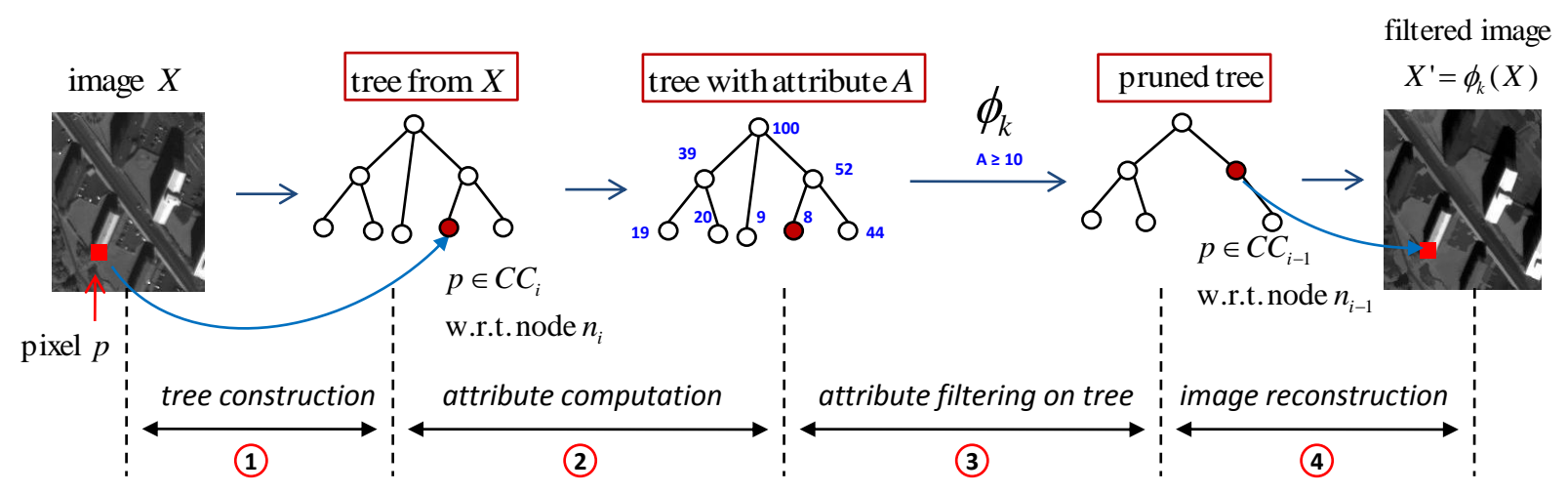

Fig. 1: The AP generation framework which involves four main stages: tree construction, attribute computation, tree-based attribute filtering (pruning) and image reconstruction from filtered (pruned) tree. This figure is adopted from [18].

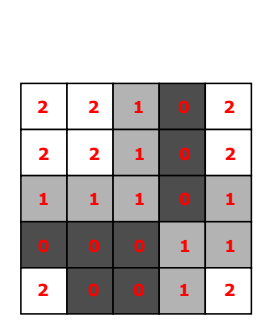

(a) Image

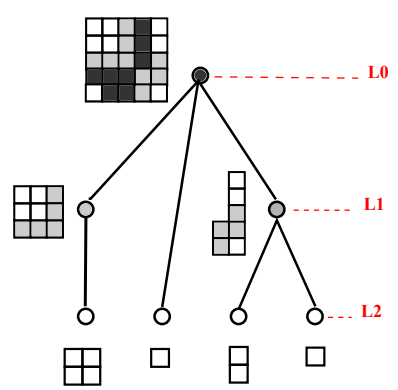

(b) Max-tree

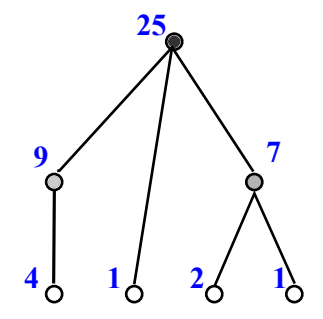

(c) Area attribute

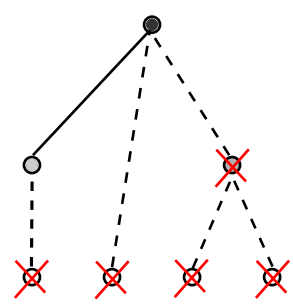

(d) Area filtering

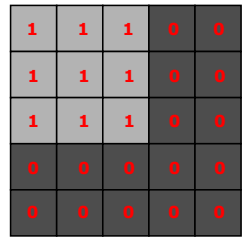

(e) Reconstruction

Fig. 2: (a) Original grayscale image $X: E \rightarrow[0,1,2]$. (b) Max-tree $T$ of $X$ computed using 4-connectivity. (c) Area (number of pixels) of the nodes of $T$. (d) Area filtering on $T$ with regard to the threshold $k=8$. (e) Image $X^{\prime}$ reconstructed from the pruned tree.

- the tree filtering rules used in the construction of APs (Sec. III-D);

- post-processing techniques intended for feature enhancement (Sec. IIII-E).

For other AP related notions, including profiles computed with different morphological filters and image reconstruction techniques, we refer readers to the related references for additional details (Sec. III-F).

\section{A. Input data}

Since APs were originally proposed to deal with only single-band images [7], their adaptation to other kinds of remote sensing data became necessary early on. In particular, their adaptation to multi-channel images (multispectral and hyperspectral) became an intensive research topic. The main idea in this regard has been to reduce the various and often correlated image bands into fewer components through some dimension reduction tool, and extract APs independently from each of them, followed by their subsequent merging.

The initial extension of APs to hyperspectral images was proposed in [19], namely Extended AP (EAP), which consists in employing the principal component analysis (PCA) to this end; an unsupervised yet often sub-optimal tool due to its linear nature. Alternatives to PCA that have been additionally studied include the independent component analysis (ICA)
[20], [21], the kernel PCA (KPCA) [22], the feature space discriminant analysis (FSDA) [23] and other supervised methods such as the discriminant analysis feature extraction (DAFE) [24], the non-parametric weighted feature extraction (NWFE) [25], the sparse Hilbert-Schmidt independence criterion and Surrogate kernel (HSIC) [26] among others.

Given that independent AP calculation from each band (or each image component, assuming dimension reduction has been applied to the image bands), ignores any and all correlational information among them, [27] have proposed a vector alternative calculating APs collectively and simultaneously from all available bands/components. The core idea relies on representing all bands through a single hierarchical representation, leading to vector APs (VAPs).

It would be also possible to compute APs on derived features of optical remote sensing data, such as edge/contour information obtained by Sobel gradient filtering of panchromatic images [10], or NDVI (Normalized difference vegetation index) from multispectral images [28] for urban and crop field classification. Then, to deal with VHR optical images where highly textural information becomes significant, another adaptation of APs was proposed in [29]. The raw input data is replaced by its textural features, thus considerably improving classification performance on the tested textured images. Furthermore, although APs are usually explored for supervised classification of optical images (either panchromatic, 
multispectral or hyperspectral), some studies have investigated them within unsupervised scenarios. For example in [30], the authors exploited the Differential APs (i.e. which compute the difference between successive APs to form differential profiles) for unsupervised anomaly detection in hyperspectral images, where they observed that the anomalies and the background of an image are enhanced in the thinning and thickening profiles, respectively. Some other examples are the retrieval of building height using panchromatic angular images [31] and the change detection in temporal panchromatic images [32].

While the application of APs to optical remote sensing data has been strongly focused on, alternative remote sensing image types have received far less attention. One may witness some tentative works on SAR (Synthetic Aperture Radar) and polarimetric SAR images for segmentation [33], building detection [34], crop field and land-cover classification [35], [36] and change detection [37]-[39] using the original APs and the Differential Attribute Profiles; on passive microwave remote sensing image analysis [40]; on LiDAR data for building detection [41] and land cover classification [16], [28], [42]-[44]; on satellite image time-series classification using Sentinel-2 data [45], [46]; on the fusion of APs and Extinction Profiles (a variant of AP that will be discussed in Sec. III-F) of hyperspectral and LiDAR data using composite kernel SVM [47], [48] and deep learning approaches [49], [50] for land cover classification. This is still an open topic for on-going and future research.

\section{B. Tree construction}

Although attribute filters have been introduced more than 20 years ago [8], the relatively late popularity of APs is mainly due to computational issues revolving around the efficient calculation of connected components, that were resolved up to a significant degree through the tree representation of images [11]. They are of paramount importance for the computation of APs, since the trees need to be computed only once and then multiple filtering outputs can be derived easily from them.

Even though the seminal work of Dalla Mura et al. [7] relies on component trees for the implementation of APs, the type of the tree is independent from the rest of the procedure, thus component trees can be replaced by alternative tree types. Consequently, and not surprisingly, there exists a number of reported works exploring such options; e.g. tree of shapes, alpha-trees and omega-trees, each with its own set of properties. Despite a plethora of tree representations for modeling connected components in mathematical morphology (of which not all have yet been implemented with the purpose of AP construction), they can be all categorized into inclusion and partitioning hierarchies. We invite interested readers to refer to the recent comprehensive survey of partition and inclusion hierarchies of images conducted by Bosilj et al. [51].

Inclusion hierarchies constitute partial partitions of a given image with nested supports and their components are formed by creating, inflating and merging image blocks [52]. Moreover, inclusion trees require the presence of a total ordering relation imposed on the set of image pixel values, which evidently renders their extension to multivariate images challenging, since ordering vectors is not straightforward.

Partitioning hierarchies on the other hand constitute full partitions of a given image, where the leaves of the hierarchy form the finest partition and are iteratively merged until a single root node is formed. In addition, partitioning hierarchies, or simply partitioning trees, most often require only a similarity metric for determining the merging or not between neighboring components, hence making them particularly suitable for processing multivariate images.

The vast majority of the reported work on APs rely on maxand min-trees that belong both to the category of inclusion trees. More formally, given a grayscale image $X: E \rightarrow$ $\mathbb{Z}, E \subseteq \mathbb{Z}^{2}$, its upper-level sets are defined as $\{X \geq t\}$ with $t \in \mathbb{Z}$ (resp. lower-level sets $\{X \leq t\}$ ), i.e. the set of images obtained by thresholding an image at all possible values of their pixels. The connected components $(C C \subseteq E)$ composing the upper or lower level sets are referred to as peak components. These two tree types (that are dual w.r.t. complementation) model the inclusion relations between these peak components, thus max-trees are excellent for modeling regions that are brighter than their surrounding, and min-trees respectively for regions that are darker. That is why besides we employ both attribute thinnings (i.e. max-tree filtering) and thickenings (i.e. min-tree filtering) during the construction of APs (Eq. (1)).

As the construction of two trees per image is both memorywise and computationally inefficient, and results in longer feature vectors per image, thus also affecting classification performance and complexity, self-dual APs (SDAPs) were introduced to target these issues [53]. More specifically, they rely on the use of the Tree of Shapes (ToS) [54], which has been designed in order to provide a unified representation for both bright and dark image structures. The ToS is constructed by filling the holes of the aforementioned peak components, and the shapes represented by the nodes do not intersect and are either disjoint or nested. Consequently, ToS is an inclusion hierarchy, which on the contrary to the component tree is also contrast-invariant and self-dual. SDAPs have been empirically shown to outperform APs consistently in terms of classification performance, while also producing shorter feature vectors per pixel [25], [53], [55].

Motivated by the success of ToS, as well as by the useful qualities of partitioning trees in this context aforementioned, $\alpha$ - and ; $\omega$-trees were recently applied to AP implementation [56]. In particular, the $\alpha$-tree is constructed based on the local range, where every tree node corresponds to an $\alpha$-connected component $(\alpha-C C)$ [57]. For instance, for $\alpha>0$, an $\alpha-C C$ is defined as the $C C$ of the maximal size such that only the neighboring pixels with gray level difference less or equal to $\alpha$ are considered connected.

Although $\alpha$-trees can lead efficiently to a complete and selfdual image representation, due to however the locality of the metric used, gray level variations within $\alpha-C C s$ can be much higher than $\alpha$ (i.e. "the chaining effect" [57]). This undesirable effect can be mitigated through the use of $\omega$-trees [58] which constitute a subset of $\alpha$-trees, constraining every $\alpha-C C$ with their global range (i.e. the maximal dissimilarity between 
any two pixels belonging to that component). The hierarchy remains self-dual, complete and capable of capturing regions of low, intermediate and high gray levels, but global range provides better grouping per level than just a local measure. Lefèvre et al. [59] have focused on the similarity metric requirement of partitioning trees for extension to multiband images, and have used metric learning in order to adapt them to hyperspectral images. Bosilj et al. [56], [60] on the other hand, have empirically tested APs, SDAPs, $\alpha$ - and $\omega$-APs against each other, and established the superiority of SDAPs in noise-free conditions, while partitioning tree based profiles outperformed their inclusion tree counterparts in terms of noise robustness.

\section{Attribute and threshold selection}

APs possess undoubtedly many desirable practical and theoretical properties that render them particularly suitable for the task of spatial-spectral description in our context. However, they are not without flaws, and the main source of their criticism so far has been in terms of their sensitivity to parameter selection [61]-[63], and by parameters we mean particularly the attributes employed to characterize every tree node, and most importantly the set of associated threshold values. The attribute and threshold selection parameters will be discussed in the subsections III-C1 and III-C2 respectively.

1) Attributes: From a theoretical point of view, any function $a: \mathcal{P}(E) \rightarrow \mathbb{R}$, where $\mathcal{P}(E)$ denotes the powerset of $E$, computable on an arbitrary collection of pixels, can be in fact employed as an attribute for AP construction. In practice, it is used during filtering by comparing a given connected component's $(C C \subseteq E)$ attribute value against a predetermined threshold in the form of a binary predicate (e.g. in case of area, "is the connected component's area greater than 300 pixels?"). It thus provides a great degree of freedom as far as the object based analysis of an image is concerned. The pioneering paper of APs [7] has introduced four such attributes: area, moment of inertia, diagonal length of bounding box and standard deviation where the first three describe a geometric property related to the shape of the tree node under study and the last, its statistical pixel intensity distribution.

Although the aforementioned four attributes are by far the most widely encountered in the state of the art, APs can accommodate (from a theoretical point of view) a vast pool of attributes. Examples include entropy, homogeneity [7], as well as the diameter of equivalent circle and area of convex hull for automatic threshold selection [64]; complexity (perimeter over area) [65]; perimeter and area of bounding box used to evaluate threshold-free APs [66]; solidity (area over area of convex hull) and orientation (between the major axis of the convex hull and the x-axis) [67]; Cov (Coefficient of variation) and NRCS (Normalized Radar Cross Section) tailored for SAR images [33], [39], where Cov is the ratio of the standard deviation divided by the mean value of pixel intensities, and NRCS, expressed in decibels, is the radar cross section per unit area of surface. Furthermore, in [68], it has been observed that when dealing with multiband input, one can extend the pool of attribute measures to include multi-dimensional functions exploiting all available bands simultaneously and two new attributes have been proposed: higher-dimensional spread and dispersion.

As far as the selection of attributes is concerned, there is no straightforward rule nor a limitation. Formally, one should use the attributes that are "meaningful" for the data under consideration; or in other words, the attributes with respect to which the objects of interest differentiate themselves from the rest. In cases where this cannot be determined or known a priori, it is "customary" to most often combine attributes that are expected to provide complementary information, e.g. some geometrical (such as area or moment of inertia) and some statistical (such as standard deviation), known as Extended Multi Attribute Profiles (EMAP) [7]. Nevertheless, in order to tackle the potentially long feature vectors resulting from the combination of various attributes, the application of dimension reduction methods is not uncommon [62].

2) Thresholds: For optimal performance of any given attribute, the set of corresponding thresholds is supposed to span the range of values between which lie the attribute values of the tree nodes representing the objects of interest. In the presence of objects of interest of varying scales however, as it is often the case in practice, the size of the threshold set (or the subdivision level of the said range) also becomes of paramount importance so as to capture their response through attribute filters. An example for illustrating this principle is the case of distinguishing minerals of various sizes with sieves; and evidently, if you only possess two sieves (one large and one very small), you cannot expect to detect minerals of inbetween sizes. Consequently, it is no surprise that the selection of the threshold set has a profound effect on the description performance of APs [27]. Although using a wide threshold range with a very fine subdivision level, might at first attempt seem as an intuitive counter-measure against this issue, it is to be avoided. Since, it will not only trigger the Hughes phenomenon, but also provides no guarantee of capturing the differences among the various scales of the objects of interest.

During the early years of APs, threshold sets were determined exclusively manually based on expert knowledge, usually with four thresholds per attribute [7], [20], [53]. Even though some attributes such as moment of inertia possess many desirable invariance properties (against scale, rotation), rendering them and their manual threshold sets robust against content variations, unfortunately most attributes are heavily affected by the spatial, spectral resolution as well as size of the objects and regions of interest.

Consequently, it was not long before attempts started being made on solving this issue. One line of research focused on developing analytical approaches based on expert knowledge, in the form of equations for producing thresholds. For instance [61], [63] have proposed such equations for area depending on the input's spatial resolution as well as for standard deviation based on the image's mean pixel intensity, where the number of thresholds however is still user-defined.

Further approaches relying on (semi-)supervised learning were put forward in [69], that proposed clustering the attributes of a given tree presentation into a user-defined number of 
clusters, while [70] investigated the evolutionary optimization of a very large set of thresholds. Then, [71], [72] presented a framework based on granulometric characteristic functions, where they employ morphological filters in order to first assess the input image's content and determine the corresponding thresholds adaptively.

More recently, unsupervised threshold selection methods [64], [73] and threshold-free APs [66], [74], [75] have been proposed. In [73], from the AP computed from all possible threshold values of an attribute, a genetic algorithm selects the subset of reconstructed images which convey the highest amount of information. A disadvantage of this technique is the expensive computation of APs for all possible threshold values. Then, a more efficient threshold selection method is proposed in [64]: first, the set of attribute values of all nodes of a component tree are sorted in non-decreasing order; then, an increasing curve is built from the sorted attribute values; finally, the points in the curve with the largest gradient values are the selected threshold values. In [74], [75] a threshold-free approach was introduced, where every tree node is described based on simple statistical properties of the sequence of attribute values belonging to the nodes in the path connecting the node under study to the root of the tree. Properties such as the highest change of attribute value have shown promise. This strategy removes the need for thresholds. Moreover, by no longer employing the same global thresholds for every node, it instead uses a node-adaptive description strategy.

\section{Tree filtering}

In terms of tree filtering, relatively few developments have taken place since the inception of APs. Nevertheless, this subsection provides an overview of filtering rules for the sake of completeness.

Tree filtering is the stage where attribute filtering is performed to a given image using its tree representation. More specifically, given an attribute and a corresponding threshold value, one removes certain nodes (either single nodes or entire branches) of the tree that do not satisfy the threshold according to some predefined strategy; such as Max, Min, Direct, Viterbi and Subtractive [7].

The effect of the selected filtering strategy depends on the increasingness (or not) of the attribute under study. Formally, an attribute $a(\cdot)$ assessed on region $C C_{i}$ is said to be increasing if the following property holds [76]:

$$
\forall C C_{1} \subseteq C C_{2}, \Rightarrow a\left(C C_{1}\right) \leq a\left(C C_{2}\right)
$$

Common increasing attributes include area and diagonal length of the bounding box. When the attribute is increasing, filtering is straightforward. More specifically, if a node does not satisfy the underlying predicate, it is removed along with all its descendants, since increasingness guarantees that they do not satisfy the predicate either. After removal, a node's pixel values become those of its highest ancestor node that satisfies the predicate. In this case, all of the aforementioned filtering strategies lead to the same exact outcome.

On the other hand, if the attribute is not increasing (e.g. moment of inertia, standard deviation, etc), then filtering is no longer straightforward, as whether a node's descendants need to be removed or not, can no longer be determined by the node under study alone. At this point one of the following is used [11]:

1) Max: prunes the nodes along a branch starting from the leaves up until the first node that satisfies the predicate and needs to be preserved.

2) Min: prunes the nodes along a branch starting from the leaves up until the last node that does not satisfy the predicate and needs to be removed.

3) Viterbi: relies on dynamic programming through the Viterbi algorithm. It formulates filtering as an optimization problem in terms of node removal and preservation costs, which solves for minimal cost.

4) Direct: consists simply in removing the nodes that do not satisfy the underlying predicate. Its eventual descendants are transferred to the first ancestor node that satisfies the predicate and thus needs to be preserved. Although simple, the direct filtering strategy is notoriously known [77] for its difficulty in dealing with shape based object analysis. This is mostly due to contrast loss related issues, which also constitutes the main motivation behind the design of the following subtractive strategy.

5) Subtractive: it behaves almost identically to the direct strategy, with the only difference being that after removing a node not satisfying the underlying predicate, an additional propagation step is performed on the descendant nodes. In particular, the pixel intensity associated with the descendant nodes is lowered in the case of max-tree (and increased in the case of min-tree), so that their contrast with respect to the local background will remain consistent once removal takes effect. In the case of tree of shapes, besides lowering the pixel intensity of the remaining descendant nodes, the subtractive rule can introduce new intensity values that were not present in the original image [55], [78].

As far as partitioning trees are concerned, the reader is referred to [56]. The subtractive strategy has been shown to outperform its alternatives when dealing with nonincreasing attributes, especially with moment of inertia [77].

The reader is referred to [55], [78] for the results of empirical comparison between filtering strategies.

\section{E. Post-processing of output profiles}

APs, i.e. the sequence of filtered images in Eq. (1), can be directly fed into supervised classifiers such as SVM or Random Forest for classification on a pixel basis. Such direct application has provided better performance compared to MPs [7] in terms of classification accuracy as well as computational cost. However, since APs often lead to feature vectors with a relatively high redundancy level, depending on the number of employed thresholds, [24], the post-processing of these features has been addressed in several studies. Many among them have proposed to apply different feature selection techniques to extract more informative features and reduce their dimension. In [24], [61], both linear (PCA, ICA) and 
nonlinear methods (ICA, KPCA, DAFE, DBFE, NWFE, etc.) have been investigated. A general framework as well as a systematic survey on spatial-spectral approaches combining APs with these feature selection techniques has been presented in [14].

Other works have focused on extra spatial processing of APs for better characterization of structural and textural information from the image content. Recent studies [79], [80] claim that when dealing with VHR remote sensing images where regions and objects appear more heterogeneous, APs may not provide a complete spatial characterization of pixels. Therefore, some efforts have been realized to improve APs through the histogram or some first-order statistical features of the local patch around the pixel under study. As a result, the local histogram-based APs (HAPs) [79], [81] and the local feature-based APs (LFAPs) [80], [82] have been proposed and proved to be more efficient for better dealing with local textures. The extensions of these extra spatial processing methods to self-dual profiles as well as to hyperspectral images have been studied in [80].

Some further notable extensions to APs include their sparse representation in an attempt to increase their description capacity [83]. In detail, through the collection of representative samples of low-dimensional class-dependent structures, any sample can then be sparsely and more effectively represented and classified. Moreover, the combination of APs with classifier ensembles has been also investigated intensively in [84], [85].

Last but not least, we have observed an increasing tendency to combine APs with convolutional neural networks $(\mathrm{CNN})$ in the classification of satellite images. While that CNNs require large training sets in order to provide optimal features from the raw data, APs can produce effective features from scratch thanks to their inherent expert knowledge. CNNs can exploit APs to produce even stronger features from them without the need for large training sets. Hence, APs simplifies the learning process of CNNs by reducing the number of training samples required for a satisfactory classification result which, consequently, reduces training time. On the downside, combining CNNs with APs increases the design and computational complexity of the classification task. For instance, we refer readers to deep learning approaches on APs [86], [87], Extinction Profiles (to be discussed in Sec. [III-F] [88] and SDAPs [89]. In those works, spatial features are extracted in two phases: first using APs (and their variants) and then using CNNs. More precisely, pixel features obtained from APs are fed into CNNs, leading to better classification results when compared to the $\mathrm{AP}$ and $\mathrm{CNN}$ methods individually, while increasing considerably the test time [86], [90].

\section{F. Extensions and generalization of APs}

As mentioned previously, the main advantages of APs in comparison to MPs are the efficient computation of APs through hierarchical image representations and the possibility of extracting information other than the ones constrained by the size and shape of structuring elements. However, using APs in remote sensing images also has a few limitations.
First, apart from threshold-free APs [66], [74], [75], the quality of an AP depends on the selected set of thresholds. A bad selection of thresholds can lead to redundant information in the AP [61], [62]. An alternative solution to alleviate this redundancy problem is to replace the attribute filters used in the computation of APs by extinction filters, resulting in Extinction Profiles (EPs) [91]. An extinction filter acts on the regional extrema (minima or maxima) of an image: each extremum is either completely preserved or pruned. Let $X$ be a grayscale image. The extinction value (with respect to a given attribute) of any maximum $M$ of $X$ is the maximum attribute value $k$ such that $M$ is still included in a maximum of $\gamma_{k}(X)$, where $\gamma_{k}$ is the thinning operator with parameter $k$. Similarly, the extinction value of any minimum $M$ of $X$ is the maximum attribute value $k$ such that $M$ is still included in a minimum of $\phi_{k}(X)$, where $\phi_{k}$ is the thickening operator with parameter $k$. This way, to compute an EP, the filtering parameter is the number of minima or maxima to be preserved instead of a threshold value, which makes EPs less sensitive to image resolution [91].

Since [91], EPs and its extension to hyperspectral images (Extended Extinction Profiles) have been successfully applied to the land cover classification of hyperspectral data [49], [92] and to the fusion of hyperspectral and Lidar data [30]. To further reduce redundancy, composite kernels are used to fuse the spatial information of EPs with hyperspectral data in [93] and with Lidar data in [47].

Another limitation of APs is that, very often, clusters of pixels associated to distinct semantic objects, such as roads and buildings, are connected by narrow paths of similar intensity value. This leads to pixels of different semantic classes being connected throughout several levels of a component tree. Consequently, the attribute values of several connected components describe the union of objects of different classes instead of an object of a single class. In [94], the authors address those problems in the context of hyperspectral image classification. To overcome those issues, attribute connected filters are replaced by partial reconstruction filters, which allows to disconnect regions connected by narrow paths and improve the overall classification accuracy. Then, this idea is explored in [43], which shows the interest of using partial reconstruction in the classification of hyperspectral and Lidar image in comparison to attribute connected filters.

Another related approach, called Invariant Attribute Profiles (IAPS), has been recently proposed in [95] to overcome other limitations of APs, including the sensibility of APs to geometric transformations, like rotation, and to the surrounding of pixels of a same material. Different from APs, IAPs are not computed from a hierarchical representation of the input data. Instead, hierarchical information is indirectly extracted from the original image by performing convolutions of different sizes and by computing the Fourier transform for different values of Fourier order. Then, IAPs are obtaining by stacking spatially invariant profiles, obtained from the segmentations of the convoluted images, and frequency invariant profiles, obtained from the histogram of oriented gradients (HOG) of the Fourier transforms.

Finally, Pham et al [96] propose a generalization of APs 
called Feature Profiles (FPS). They generalize the step 4) of the generation of APs, which consists in reconstructing an image from a filtered tree. To build an AP, this reconstruction is originally performed by projecting the gray values of the nodes of the filtered tree onto the image pixels. In [96], this reconstruction step is extended by taking into consideration not only the gray values of the nodes, but also other statistical and geometrical features. The resulting images compose the so called FP. The experiments with remote sensing images of [96], [97] show the interest of projecting attributes like area and moment of inertia in the context of image classification. Hence, we also consider FPs later in the experimental section.

\section{EXPERIMENTAL STUDY}

This section describes our experimental study to evaluate the performance of the standard APs as well as some of their recent variants. The contributions of this section are two-fold: the evaluation of newer variants of APs, and of the impact of connectivity and quantization parameters on the performance of APs. This is the first study addressing the connectivity and quantization parameters' effect in this context.

Experiments were mostly performed in Python using publicly available libraries 3 First, APs and some of their variants were computed with the Simple Attribute Profile (SAP) package 4 . To the best of our knowledge, this is the first available open-source library for computing APs and some of their extensions. The SAP package relies on the Higra [98] library 5 , which provides efficient implementation and postprocessing of morphological trees in $\mathrm{C}++$. Then, classification was performed with the scikit-learn Python library.

Supervised classification has been conducted on both grayscale and hyperspectral images for the sake of comprehensiveness. In this section, we introduce the datasets, the experimental setup commonly encountered in the state of the art [7], [14], and the classification results that have been obtained.

In more detail, the standard setup involves calculating the hierarchical tree representation (as explained in detail at Section (II) from the entire input image, computing the APs (or its variants) from it, and then subdividing the resulting features based on the locations of training and validation/testing pixels. The next section will elaborate on the reasons why this approach constitutes a validation malpractice and propose an alternative strategy for better assessing the generalization capacity of APs.

\section{A. Data description}

The experiments have been conducted with two publicly available datasets for the sake of reproducibility. In order to show performance variations depending on the number of spectral bands, one hyperspectral and one panchromatic dataset have been selected.

${ }^{3}$ Source codes are available in https://gitlab.inria.fr/dsantana/attributesprofiles-survey-source-codes

${ }^{4}$ The documentation and source codes of the SAP package are provided in https://gitlab.inria.fr/fguiotte/sap

${ }^{5}$ The documentation and source codes of the Higra package are provided in https://github.com/higra

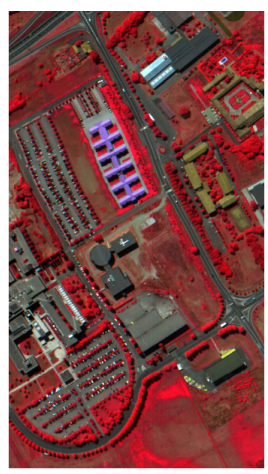

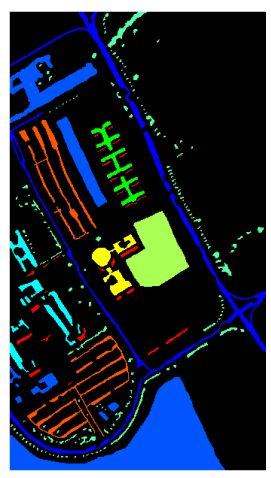

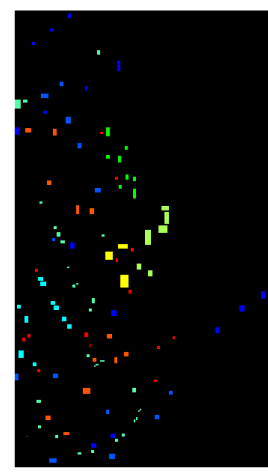

Thematic classes:
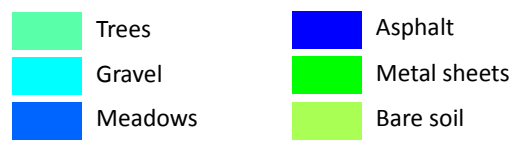

Bitumen

Shadows

Self-blocking bricks
Fig. 3: The $610 \times 340$ Pavia University data (left to right: falsecolor image made by bands 31-56-102, ground truth including nine thematic classes and training set).

1) Pavia University dataset: The Pavia dataset is a hyperspectral image acquired by the ROSIS airborne sensor with 1.3-m spatial resolution over the region of Pavia University, Italy 6 The image consists of $610 \times 340$ pixels with 103 spectral bands (from 0.43 to $0.86 \mu \mathrm{m}$ ) after the noisy bands are removed. The ground truth covers nine thematic classes: trees, asphalt, bitumen, gravel, metal sheets, shadows, meadows, self-blocking bricks and bare soil. For this image, the standard training ser7 composed of 3,921 pixels (see Tab. I), was adopted for the classification task. The test set was composed of all the remaining 40,002 pixels in the ground truth that are not in the training set. In the remainder of this paper, this partition into training/test set of Pavia will be denoted as Pavia $_{1}$. The reader may note that some works in the literature consider all ground truth pixels as testing pixels, which is not done here in order to provide fair classification results with a non-void intersection between training and testing pixels. The false-color image (made by combining the bands 31, 56 and 102), the ground truth map and the training set are shown in Fig. 3. Following the standard approach of handling hyperspectral images [7], we first performed the PCA on this dataset and the first four PCs (involving more than $99 \%$ of the total variance) were preserved for our experiments. The APs and their extensions are computed independently on each selected PCs and are concatenated, leading to EAPs. This way, we assess the family of EAPs and its variants on the Pavia dataset.

2) Gray-Potsdam dataset: The Potsdam dataset is composed of 38 aerial high resolution images, of $6000 \times 6000$ pixels, with $5 \mathrm{~cm}$ spatial resolution over the city of Pots-

${ }^{6}$ The Pavia dataset and its ground truth can be downloaded in http://www.ehu.eus/ccwintco/index.php/Hyperspectral_Remote_Sensing_Scenes

${ }^{T}$ Training set provided by the IEEE Geoscience and Remote Sensing Society (GRSS) Data and Algorithm Standard Evaluation (DASE) website: http://dase.grss-ieee.org/ 
TABLE I: Number of training and test samples of the standard $\left(\right.$ Pavia $\left._{1}\right)$ and new (Pavia $)$ partitions of the Pavia dataset.

\begin{tabular}{l|rrr}
\hline \hline \multirow{2}{*}{ Class } & \multicolumn{3}{|c}{ Number of samples } \\
\cline { 2 - 4 } & Training set & Test set & Total \\
\hline Asphalt & 548 & 6,304 & 6,852 \\
Meadows & 540 & 18,146 & 18,686 \\
Gravel & 392 & 1,815 & 2,207 \\
Trees & 524 & 2,912 & 3,436 \\
Metal sheets & 265 & 1,113 & 1,378 \\
Bare Soil & 532 & 4,572 & 5,104 \\
Bitumen & 375 & 981 & 1,356 \\
Bricks & 514 & 3,364 & 3,878 \\
Shadows & 231 & 795 & 1,026 \\
\hline \multicolumn{3}{l}{}
\end{tabular}

dam, Germany 8 For each image, the red, green, blue and infrared bands are available. The ground-truth annotations of this dataset cover six thematic classes including impervious surfaces, building, low vegetation, tree, car, and clutter/background (water bodies, tennis courts, swimming pools, etc). Experiments were performed on one image of this dataset, namely top_potsdam_7_7.tiff, whose ground truth is composed of several connected components of each of the six classes. To highlight the strength of the spatial information extracted from APs in the context of image classification, we considered a grayscale version of the original RGB image. The original RGB image was converted into a grayscale image using the formula $0.3 R+0.59 G+0.11 B$, which gives an approximation of the luminance in the NTSC color space [99]. The input grayscale image together with its thematic ground truth map are shown in Fig. 4(a) and 4(b), respectively. To prevent biased results towards the majority class, ten training sets were obtained by random sampling the same number of pixels from each of the six thematic classes (see Table II). In total, for each random split, 360,000 pixels (1\% of the groundtruth samples) were selected for training and the remaining $35,640,000$ pixels were used for testing. In the remainder of this paper, those random partitions into training/test set of Gray-Potsdam will be denoted as GrayPotsdam . Due to the dimensions of Gray-Potsdam, the random training pixels of GrayPotsdam 1 cannot be visualized when the image is downsized. The reader can refer to the source codes of our experiments 9 to visualize the train/test splits of the GrayPotsdam dataset.

TABLE II: Number of training and test samples per class of the partitions GrayPotsdam 1 and GrayPotsdam 2 of the GrayPotsdam dataset.

\begin{tabular}{l|rrr}
\hline \hline \multirow{2}{*}{ Class } & \multicolumn{3}{|c}{ Number of samples } \\
\cline { 2 - 4 } & Training set & Test set & Total \\
\hline Background & 60,000 & $1,565,250$ & $1,625,250$ \\
Trees & 60,000 & $5,785,203$ & $5,845,203$ \\
Cars & 60,000 & 631,810 & 691,810 \\
Buildings & 60,000 & $12,362,473$ & $12,422,473$ \\
Low vegetation & 60,000 & $8,639,455$ & $8,699,455$ \\
Impervious surfaces & 60,000 & $6,655,809$ & $6,715,809$ \\
\hline
\end{tabular}

${ }^{8}$ The Potsdam dataset and its ground truth can be downloaded from http://www2.isprs.org/commissions/comm3/wg4/2d-sem-label-potsdam.html

${ }^{9}$ Source codes are available in https://gitlab.inria.fr/dsantana/attributesprofiles-survey-source-codes

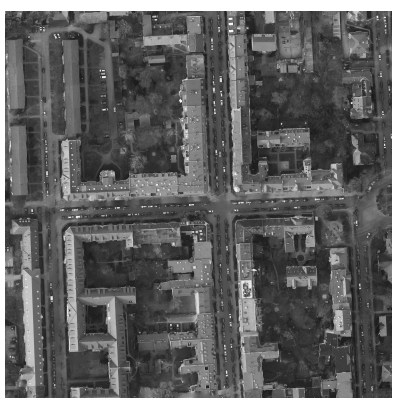

(a)

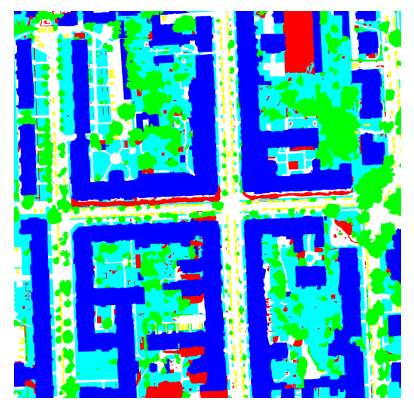

(b)

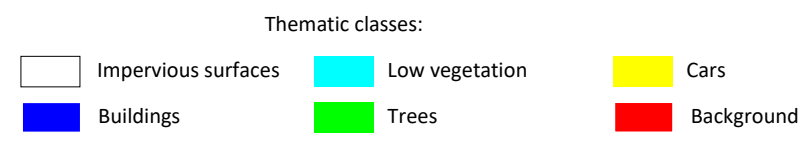

Fig. 4: Grayscale version of the image top_potsdam_7_7.tiff of the Potsdam dataset (a) and its thematic ground truth (b).

\section{B. Experimental setup}

The experiments have been conducted using a wide variety of parameters, shown in Table III. Some of which have been employed for the first time in the state of the art. To ease the readability of the results presented in this section and in the following section, we present a summary of our experimental settings in tables IV and $\mathrm{V}$. Tab. IV shows the parameter settings used for each experiment, and Tab. $\mathrm{V}$ contains the description of each dataset split employed in our experiments.

The popular choices of classifiers for APs are SVM and Random Forest (RF) [14]. As suggested in [14], RFs usually perform better than SVMs in this context. Moreover, RFs require lower training and prediction time [80]. Hence, our supervised classification was conducted on the two datasets using the RF classifier [100] with 100 trees, as employed in other studies [19], [96]. In the scikit-learn implementation of random forests, a random shuffling of the data is applied before training. Hence, the classification output for each run is not reproducible unless a fixed value is assigned to the random_state parameter of the RandomForestClassifier class. To take this into consideration, the classification results reported in this section and in the following section are the average and the standard deviation of the scores obtained for ten runs on each dataset. The number of variables involved in the training was set to the square root of the feature vector length. In order to evaluate and compare classification accuracy of different approaches, overall accuracy (OA), average accuracy (AA), and kappa coefficient $(\kappa)$ have been taken into account [7]. As far as attribute filtering is concerned, for the sake of design simplicity and computational cost we have limited our experiments with two attributes, area and the moment of inertia. The former is increasing and the latter is not. For Pavia dataset, fourteen area thresholds were computed automatically using the formula proposed in [61]: 
TABLE III: Choice of methods and parameters employed in the reported tests.

\begin{tabular}{ccccccc}
\hline \hline Datasets & Attributes & Tree Types & Quantization & Connectivity & Post-processing & Thresholds \\
\hline PaviaU & Area & Max-Min & 8 bits & 4 & HAPs & manual \\
Gray-Potsdam & Moment of inertia & Tree of shapes & 16 bits & 8 & FPs & automatic \\
& & $\alpha$-tree & 64 bits & & & LFAPs \\
& & $\omega$-tree & & & \\
& Max-tree & & & \\
& & Min-tree & & & \\
& & &
\end{tabular}

TABLE IV: Experimental settings employed in the reported tests.

\begin{tabular}{|c|c|c|c|c|c|c|c|c|c|}
\hline & & & Dataset split & Attributes & Tree types & Quantization & Connectivity & Post-Processing & Thresholds \\
\hline Sec. IV & Tab. & $\mathrm{VI}$ & Pavia $_{1}$ & All & All & 8 bits & 4 & All & All \\
\hline Sec. IV & Tab. & VIII & GrayPotsdam $_{1}$ & All & All & 8 bits & 4 & $\begin{array}{c}\text { FPs } \\
\text { LFAPs }\end{array}$ & $\begin{array}{l}\text { manual } \\
\text { without }\end{array}$ \\
\hline Sec. IV & Tab. & $\mathrm{X}$ & GrayPotsdam $_{1}$ & All & All & 8 bits & $\begin{array}{l}4 \\
8 \\
\end{array}$ & $\begin{array}{c}\text { FPs } \\
\text { LFAPs }\end{array}$ & $\begin{array}{l}\text { manual } \\
\text { without }\end{array}$ \\
\hline Sec. IV & Tab. & XI & Pavia $_{1}$ & All & All & 8 bits & $\begin{array}{l}4 \\
8 \\
\end{array}$ & All & All \\
\hline Sec. IV & $\mathrm{Tab}$. & XII & Pavia $_{1}$ & All & All & $\begin{array}{c}8 \text { bits } \\
16 \text { bits } \\
32 \text { bits }\end{array}$ & 4 & All & All \\
\hline Sec. V & Tab. & $\mathrm{XIII}$ & $\begin{array}{l}\text { Pavia }_{1} \\
\text { Pavia }_{2} \\
\end{array}$ & All & All & 8 bits & 4 & FPs & All \\
\hline Sec. V & Tab. & XIV & Pavia $_{2}$ & All & All & 8 bits & 4 & FPs & All \\
\hline Sec. V & Tab. & $\mathrm{XV}$ & $\begin{array}{l}\text { GrayPotsdam }_{1} \\
\text { GrayPotsdam }_{2}\end{array}$ & All & All & 8 bits & 4 & FPs & $\begin{array}{l}\text { manual } \\
\text { without }\end{array}$ \\
\hline Sec. $V$ & Tab. & $\mathrm{XVI}$ & Potsdam $_{2}$ & All & All & 8 bits & 4 & FPs & $\begin{array}{l}\text { manual } \\
\text { without }\end{array}$ \\
\hline Sec. V & Tab. & XVII & Potsdam $_{3}$ & All & All & 8 bits & 4 & FPs & manual \\
\hline Sec. V & Tab. & XVIII & Potsdam $_{3}$ & All & All & 8 bits & 4 & FPs & manual \\
\hline
\end{tabular}

TABLE V: Pavia and Gray-Potsdam dataset splits employed in the reported tests.

\begin{tabular}{|c|c|c|}
\hline Dataset split & Description & Objective \\
\hline Pavia $_{1}$ (Fig. 3 ) & $\begin{array}{l}\star \text { Standard split obtained from http://dase.grss-ieee.org/ } \\
\star \text { Commonly used in the literature. }\end{array}$ & $\begin{array}{l}\star \text { Provide evaluation scores of AP extensions which are } \\
\text { comparable with the results in the literature (in which the } \\
\text { whole data is preprocessed with PCA, and training/testing } \\
\text { features are extracted from the same tree, leading to the } \\
\text { 'leakage' of training features). }\end{array}$ \\
\hline Pavia $_{2}$ (Fig. 9 b)) & $\begin{array}{l}\star \text { Ten sets of training/test samples randomly extracted from } \\
\text { a restricted region of the Pavia data. } \\
\star \quad \text { Lower variability of training pix- } \\
\text { els when compared to Pavia } \\
\star \text { Better separation between traning/testing pixels than } \\
P_{\text {Pavia }} \text {. } \\
\star \text { Same number of training samples per class as Pavia }\end{array}$ & $\begin{array}{l}\star \text { Evaluate the impact of having large ground-truth regions } \\
\text { not contributing to the training set. } \\
\star \text { Generalize APs to datasets with lower levels of 'leakage' } \\
\text { of training features, which still remain due to the whole data } \\
\text { being preprocessed with PCA, and to training and testing } \\
\text { features being extracted from the same tree. } \\
\star \text { Provide a more realistic partition of the Pavia University } \\
\text { dataset when compared to Pavia. }\end{array}$ \\
\hline GrayPotsdam $_{1}$ & $\begin{array}{l}\star \text { Ten sets of training/test samples randomly extracted from } \\
\text { all of the ground-truth connected components of Gray- } \\
\text { Potsdam. } \\
\star \text { Same number of training samples per semantic class. }\end{array}$ & $\begin{array}{l}\star \text { Evaluate APs and its extensions on Gray-Potsdam using } \\
\text { the training/test splitting method commonly used in the } \\
\text { literature. }\end{array}$ \\
\hline GrayPotsdam $_{3}$ (Fig. П]) & $\begin{array}{l}\star \text { Dataset divided in half. } \\
\star \text { Same number of training pixels per class as } \\
\text { GrayPotsdam } 1 \text { and GrayPotsdam } 2 \text {, but different } \\
\text { number of test pixels. } \\
\star \text { Test set composed of all pixels from the lower half. } \\
\star \text { Ten sets of training samples randomly extracted from the } \\
\text { upper half. }\end{array}$ & $\begin{array}{l}\star \text { Generalize APs to multiple-image datasets. } \\
\star \text { Compute training and testing features from different trees } \\
\text { obtained from the two halves of the image, with no leakage } \\
\text { of training/testing features. }\end{array}$ \\
\hline
\end{tabular}




$$
\lambda_{a}, X=\frac{1000}{v}\left\{a_{\min }, a_{\min }+\delta_{a}, a_{\min }+2 \delta_{a}, \ldots, a_{\max }\right\}
$$

that has received wide acclaim in related works [14], [27], [96], [101]. In Eq. (3) $a_{\min }$ and $a_{\max }$ are initialized by 1 and 14 respectively, with a step increase $\delta_{a}$ equal to 1 and $v$ represents the spatial resolution of the input data. The resulting thresholds for the Pavia dataset follow:

$$
\begin{array}{r}
\lambda_{a, \text { Pav }}=\{770,1538,2307,3076,3846,4615,5384, \\
6153,6923,7692,8461,9230,10000,10769\} .
\end{array}
$$

For the Gray-Potsdam dataset, the thresholds obtained with the previous formula were not sufficient to cover the size variations of the targeted classes. To obtain a set of thresholds spanning a larger range of values without increasing the number of thresholds, we considered the fourteenth first values of the geometric sequence whose $n$-th term is given by $200 \times 2^{n}$. The resulting thresholds for the Gray-Potsdam follow:

$$
\lambda_{a, P o t}=\{200,400,800,1600,3200,6400,12800,
$$

$25600,51200,102400,204800,409600,819200,1638400\}$.

As far as moment of inertia is concerned, the manually set thresholds used in several studies [19], [25], [27] were adopted here as well:

$$
\lambda_{i, \text { Pot }}=\lambda_{i, \text { Pav }}=\{0.2,0.3,0.4,0.5\} .
$$

Moreover, as mentioned in Tab. III] we consider two additional experimentation parameters, namely the input image's connectivity and quantization level, the effect of which, to the best of our knowledge, has not been previously studied in the state of the art. For instance in [49], [91], [102], the authors use 4-connectivity, but in most published studies these two parameters are seldom mentioned.

For the sake of simplicity and to avoid introducing new acronyms, EAP will be referred to as AP in the remainder of this article. Hence, whenever we discuss results on the Pavia dataset, it should be understood that the APs and its variants corresponds to EAPs on this dataset.

In more detail, we compare APs and EAPs generated from different kinds of trees including: max-tree (AP-maxT), mintree (AP-minT), a max-tree along with a min-tree (as in standard APs) [7], the SDAPs [53] from tree of shapes, the $\alpha$-APs and $\omega$-APs from $\alpha$ - and $\omega$-trees respectively [56]. We also provide the results of some effective post-processing techniques including the HAPs/HSDAPs [79], [80], LFAPs/LFSDAPs [80] and of some extensions of APs, including Threshold-Free (TF) APs [74] and FPs [96]. A far more limited comparative study of these parameters is reported in [97]. We obtained HAPs and HSDAPs using histograms of 7 bins and a window size of $7 \times 7$ pixels, which are the optimal parameter settings according to the experiments of [96]. LFAPs and LFSDAPs were computed by using the mean and the standard deviation of $7 \times 7$ sized windows as the local features. As shown in [96], feature and histogram profiles are fairly robust to the choice of window size, but the experiments of [96] with
$7 \times 7$ windows provided superior results. FPs were obtained by projecting the average gray levels $\left(\mathrm{FP}_{\mu}\right)$, the area $\left(\mathrm{FP}_{a}\right)$ and both average gray levels and area $\left(\mathrm{FP}_{\mu+a}\right)$ of tree nodes during the reconstruction step, as done in [96]. Among all tested methods, TF AP [74] is the only one not implemented in the SAP library.

\section{Results}

This subsection will start with the presentation and discussion of results obtained from experiments employing the commonly encountered 8-bit quantization and 4-connectivity [49], [91], [102] in the state of the art. Then, it will continue with an evaluation of the effect of the aforementioned two parameters on classification performance.

1) Classification results for Pavia dataset: The overall classification results, the classification results per class and the classification maps for the Pavia dataset obtained using the partition Pavia $_{1}$ are presented in Tab. VI Tab. VII and Fig. 5. respectively.

For this dataset, the tree type underlying the APs appears to have an important influence on performance. More specifically, the $\alpha$-APs and $\omega$-APs outperformed both APs and SDAPs. In particular, by using the $\omega$-tree, one can achieve an average OA of $96.33 \%$, i.e. $5.76 \%$ and $2.23 \%$ better than standard APs and SDAPs, respectively. However, this improvement is not observed for all classes. While the accuracy of $\omega$-AP for the gravel class increases by $20.58 \%$ with respect to APs, the accuracy for the asphalt class decreases by $5.98 \%$. Then, by post-processing the AP and the SDAP with histogram and local feature profiles, we improved AP (resp. SDAP) by more than $4 \%$ (resp. 1\%) in terms of overall accuracy. Regarding the feature profiles, $\mathrm{FP}_{\mu}$ and $\mathrm{FP}_{\mu+a}$ provided much better results than $\mathrm{FP}_{a}$, as already shown in the original paper on FPs [96]. Finally, the threshold-free profile, which has less dimensions than all other tested profiles, outperformed AP by $3.41 \%$, $3.13 \%$ and $4.51 \%$ in terms of OA, AA and $\kappa$, respectively. Among all tested methods, the best classification accuracy was achieved by $\mathrm{FP}_{\mu}$, with $\mathrm{OA}=96.76$ and $\kappa \times 100=95.65$. Compared to the standard APs, an enhancement of $6.19 \%$ in $\mathrm{OA}$ and $8.12 \%$ in $\kappa$ was adopted.

Furthermore, our classification results on the Pavia dataset are competitive with respect to some recent deep learning approaches discussed in [17]. In [17], the authors compare several CNN architectures from the literature for hyperspectral image classification. Among the tested architectures, the best results for Pavia $(O A=84.32 \pm 0.72$ and $\kappa=0.799 \pm 0.009)$, obtained with the same dataset split ( Pavia $_{1}$ ) employed here, achieved with a 3D CNN [103], are still inferior to most of our results given in Tab. VI

The reader may note that our classification results differ from the ones presented in the original papers of (extended) APs [19], SDAPs [25], LFAPS [80], $\alpha-$ and $\omega$-APs [56]. More precisely, the differences can be explained by the various sets of attributes and thresholds employed in some of the papers [19], [25], [80], by the use of pre- and post-processing techniques other than PCA [25], and by number of RF trees [25]. Moreover, even using the same set of thresholds and the 
TABLE VI: Classification result of Pavia dataset obtained by different methods using the default 4-connectivity and 1-byte quantization.

\begin{tabular}{|c|c|c|c|c|}
\hline \multirow{2}{*}{ Method } & \multirow{2}{*}{ Dimension } & \multicolumn{3}{|c|}{ Classification result } \\
\hline & & $\mathrm{OA}(\%)$ & AA (\%) & $\kappa \times 100$ \\
\hline $4 \mathrm{PC}$ & 4 & $65.27 \pm 0.25$ & $74.88 \pm 0.20$ & $56.93 \pm 0.27$ \\
\hline AP-maxT & 80 & $89.21 \pm 0.63$ & $87.37 \pm 0.19$ & $85.42 \pm 0.80$ \\
\hline AP-minT & 80 & $87.11 \pm 2.00$ & $92.62 \pm 0.61$ & $83.23 \pm 2.41$ \\
\hline AP & 152 & $90.57 \pm 2.60$ & $93.13 \pm 0.67$ & $87.53 \pm 3.32$ \\
\hline SDAP & 80 & $94.10 \pm 0.21$ & $93.85 \pm 0.37$ & $92.16 \pm 0.27$ \\
\hline$\alpha-\mathrm{AP}$ & 80 & $95.46 \pm 0.59$ & $95.25 \pm 1.23$ & $93.91 \pm 0.79$ \\
\hline$\omega$-AP & 80 & $96.33 \pm 0.40$ & $\mathbf{9 7 . 1 7} \pm \mathbf{0 . 8 9}$ & $95.08 \pm 0.54$ \\
\hline HAP & 1064 & $94.73 \pm 0.30$ & $92.96 \pm 0.31$ & $92.84 \pm 0.42$ \\
\hline HSDAP & 340 & $95.35 \pm 0.29$ & $94.00 \pm 0.49$ & $93.79 \pm 0.39$ \\
\hline LFAP & 304 & $94.75 \pm 0.29$ & $94.12 \pm 0.41$ & $92.97 \pm 0.39$ \\
\hline LFSDAP & 160 & $96.34 \pm 0.16$ & $92.81 \pm 0.20$ & $95.06 \pm 0.22$ \\
\hline $\mathrm{FP}_{\mu}$ & 152 & $96.76 \pm \mathbf{0 . 1 5}$ & $97.05 \pm 0.19$ & $95.65 \pm 0.21$ \\
\hline $\mathrm{FP}_{a}$ & 152 & $85.48 \pm 0.98$ & $93.94 \pm 0.55$ & $81.36 \pm 1.16$ \\
\hline $\mathrm{FP}_{\mu+a}$ & 304 & $96.15 \pm 0.14$ & $96.74 \pm 0.32$ & $94.82 \pm 0.19$ \\
\hline TF-AP & 72 & $93.98 \pm 0.53$ & $96.26 \pm 0.29$ & $92.04 \pm 0.68$ \\
\hline
\end{tabular}

TABLE VII: Classification results per class of Pavia dataset obtained by different methods using the default 4-connectivity and 1-byte quantization.

\begin{tabular}{|c|c|c|c|c|c|c|c|c|c|c|}
\hline \multirow{2}{*}{ Method } & \multirow{2}{*}{ Dimension } & \multicolumn{9}{|c|}{ Accuracy per class (\%) } \\
\hline & & Asphalt & Meadow & Gravel & Tree & Metal & Soil & Bitumen & Brick & Shadow \\
\hline $4 \mathrm{PC}$ & 4 & $71.25 \pm 0.44$ & $53.20 \pm 0.45$ & $38.39 \pm 0.53$ & $98.28 \pm 0.10$ & $98.79 \pm 0.17$ & $67.34 \pm 0.58$ & $66.74 \pm 0.96$ & $83.28 \pm 0.44$ & $96.64 \pm 0.34$ \\
\hline AP-maxT & 80 & $92.33 \pm 0.14$ & $93.66 \pm 1.41$ & $43.32 \pm 0.67$ & $95.61 \pm 0.77$ & $99.63 \pm 0.08$ & $71.07 \pm 0.24$ & $97.83 \pm 0.14$ & $95.28 \pm 0.13$ & $97.61 \pm 0.46$ \\
\hline AP-minT & 80 & $92.81 \pm 0.08$ & $80.28 \pm 4.42$ & $86.02 \pm 1.65$ & $98.97 \pm 0.08$ & $99.90 \pm 0.08$ & $84.19 \pm 0.08$ & $99.93 \pm 0.12$ & $98.02 \pm 0.13$ & $93.47 \pm 3.01$ \\
\hline AP & 152 & $95.71 \pm 0.21$ & $87.38 \pm 5.81$ & $73.17 \pm 3.00$ & $99.09 \pm 0.29$ & $99.65 \pm 0.05$ & $85.64 \pm 0.14$ & $\mathbf{1 0 0 . 0} \pm \mathbf{0 . 0 0}$ & $99.24 \pm 0.18$ & $98.25 \pm 2.03$ \\
\hline SDAP & 80 & $97.15 \pm 0.28$ & $92.51 \pm 0.47$ & $77.15 \pm 0.29$ & $93.21 \pm 0.70$ & $99.83 \pm 0.06$ & $99.08 \pm 0.00$ & $98.94 \pm 0.25$ & $98.15 \pm 0.50$ & $88.62 \pm 3.07$ \\
\hline$\alpha-\mathrm{AP}$ & 80 & $89.39 \pm 0.21$ & $96.63 \pm 0.43$ & $76.27 \pm 11.05$ & $99.73 \pm 0.07$ & $99.61 \pm 0.07$ & $98.93 \pm 0.06$ & $99.49 \pm 0.00$ & $99.35 \pm 0.10$ & $97.82 \pm 0.44$ \\
\hline$\omega-\mathrm{AP}$ & 80 & $89.73 \pm 0.56$ & $96.70 \pm 0.42$ & $93.75 \pm 7.84$ & $99.75 \pm 0.09$ & $99.64 \pm 0.04$ & $98.95 \pm 0.06$ & $99.49 \pm 0.00$ & $99.36 \pm 0.11$ & $97.12 \pm 0.40$ \\
\hline HAP & 1064 & $99.86 \pm 0.16$ & $97.68 \pm 0.42$ & $63.83 \pm 1.71$ & $97.74 \pm 0.11$ & $99.96 \pm 0.06$ & $80.73 \pm 2.22$ & $99.99 \pm 0.03$ & $98.13 \pm 0.07$ & $98.73 \pm 0.33$ \\
\hline HSDAP & 340 & $99.70 \pm 0.18$ & $94.49 \pm 0.46$ & $68.59 \pm 2.44$ & $95.25 \pm 0.19$ & $99.99 \pm 0.03$ & $99.68 \pm 0.39$ & $99.55 \pm 0.10$ & $99.08 \pm 0.06$ & $89.70 \pm 2.48$ \\
\hline LFAP & 304 & $90.33 \pm 0.90$ & $96.23 \pm 0.36$ & $72.68 \pm 3.79$ & $96.79 \pm 0.10$ & $99.20 \pm 0.24$ & $98.08 \pm 0.09$ & $99.36 \pm 0.17$ & $97.28 \pm 0.10$ & $97.12 \pm 0.79$ \\
\hline LFSDAP & 160 & $99.42 \pm 0.18$ & $98.42 \pm 0.12$ & $65.82 \pm 0.51$ & $92.88 \pm 0.80$ & $99.52 \pm 0.18$ & $97.06 \pm 0.56$ & $99.16 \pm 0.14$ & $98.75 \pm 0.13$ & $84.26 \pm 1.25$ \\
\hline $\mathrm{FP}_{\mu}$ & 152 & $94.97 \pm 0.13$ & $97.10 \pm 0.21$ & $88.65 \pm 0.91$ & $97.07 \pm 0.19$ & $99.89 \pm 0.04$ & $98.43 \pm 1.57$ & $100.0 \pm 0.00$ & $97.43 \pm 0.53$ & $99.92 \pm 0.06$ \\
\hline $\mathrm{FP}_{a}$ & 152 & $95.51 \pm 0.25$ & $73.87 \pm 2.20$ & $92.31 \pm 4.14$ & $99.31 \pm 0.20$ & $99.93 \pm 0.04$ & $86.98 \pm 1.18$ & $99.98 \pm 0.04$ & $99.72 \pm 0.10$ & $97.80 \pm 1.82$ \\
\hline $\mathrm{FP}_{\mu+a}$ & 304 & $96.03 \pm 0.23$ & $96.61 \pm 0.21$ & $92.43 \pm 1.28$ & $97.93 \pm 0.43$ & $99.89 \pm 0.04$ & $91.00 \pm 0.96$ & $99.97 \pm 0.05$ & $98.57 \pm 0.38$ & $98.23 \pm 1.78$ \\
\hline TF-AP & 72 & $95.79 \pm 0.26$ & $90.31 \pm 1.14$ & $82.42 \pm 2.41$ & $99.64 \pm 0.08$ & $99.75 \pm 0.10$ & $99.23 \pm 0.19$ & $100.0 \pm \mathbf{0 . 0 0}$ & $99.61 \pm 0.06$ & $99.56 \pm 0.06$ \\
\hline
\end{tabular}

same number of RF trees, as done in [56], the quantization and connectivity parameters, which are not explicitly given in those papers, may play a role in the final results, as discussed later in Sec. IV-D Nevertheless, in term of conclusion, there is no incoherence between our paper and the papers cited in the beginning of this paragraph.

2) Classification results for Gray-Potsdam dataset: The overall and per-class classification results for the GrayPotsdam dataset using GrayPotsdam 1 are presented in Tables VIII and IX, respectively. As already mentioned previously, the reported results are the average scores over ten runs on the different random training-test splits of GrayPotsdam . $_{\text {. }}$ Since LFAPs perform better than HAPs in general, as attested by [80], and due to the expensive computation of HAPs, we consider only LFAPs in our experiments with the GrayPotsdam dataset.

In the case of the Gray-Potsdam dataset, it can be observed that AP variants boost accuracy consistently at various degrees. In particular, the $\alpha$-APs and $\omega$-APs could outperform APs on each single max-tree or min-tree but still falls below the standard APs. On the other hand, SDAP performed better than AP, $\alpha$-AP and $\omega$-AP. Then, by post-processing the output profiles, LFAP and LFSDAP outperformed AP and SDAP by $3.52 \%$ and $4.61 \%$, respectively, in terms of overall accuracy. Among the feature profiles, the best result was achieved by
$\mathrm{FP}_{\mu+a}$, which outperforms the APs by more than $2 \%$ in terms of $O A, A A$ and $\kappa$. Finally, the threshold-free AP presented lower scores than APs, but it outperformed AP-maxT, APminT, $\alpha$-AP and $\omega$-AP despite having the smallest number of dimensions among all methods. In conclusion, the best classification result was obtained by LFSDAP with $80.80 \%$ of overall accuracy, which represents an improvement of $5.70 \%$ with respect to the standard AP.

Fig. 6(a) and (b) present a crop of the Gray-Potsdam dataset and of its ground-truth, respectively, which are composed of the first 500 lines (from the top to the bottom) and 1200 columns (from left to right) of the original data. Fig. 6(c)(o) illustrates the classification maps obtained on the crop of Fig. 6(a) using the aforementioned methods. We can see that the classification based solely on pixels gray value is very noisy in most regions of the image. By incorporating spatial information from attribute profiles, we see a more structured result, with a clearer separation between the regions of different classes. Furthermore, the post-processing of APs and SDAPs with local features successfully reduces the noise in all classes, especially the regions containing trees (in green) and buildings (in blue).

In terms of future research directions, it would be interesting to investigate the combination potential of the AP variants and extensions, in an effort to discover whether they provide com- 


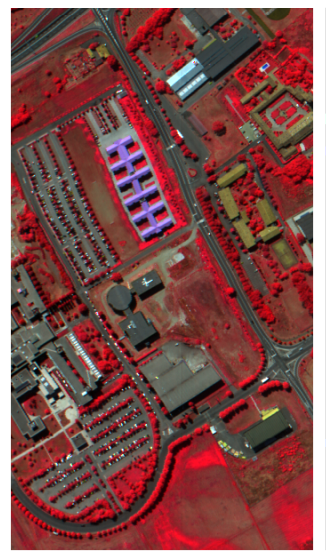

(a) Image

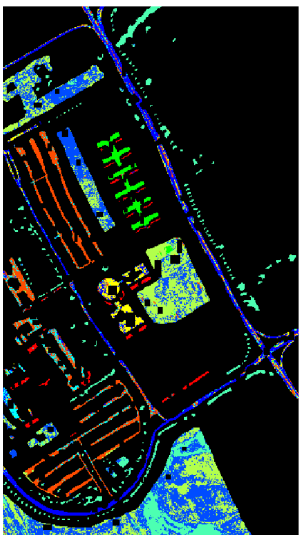

(d) 4 PCs

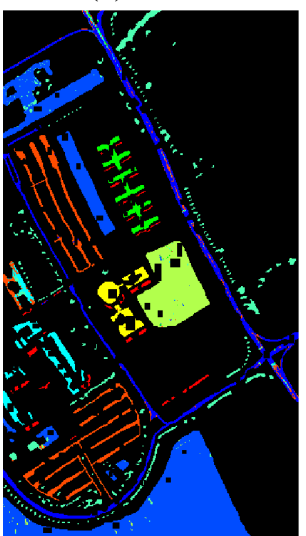

(i) $\alpha$-AP

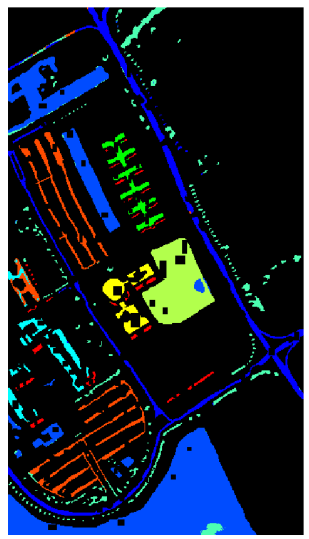

(n) HSDAP

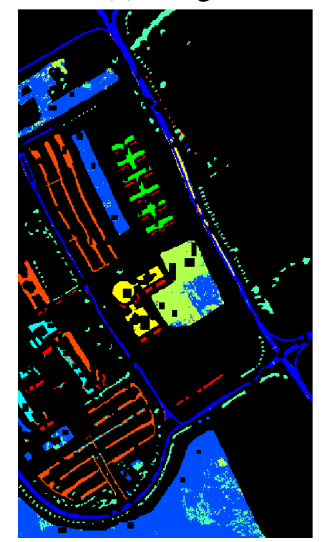

(e) AP-maxT

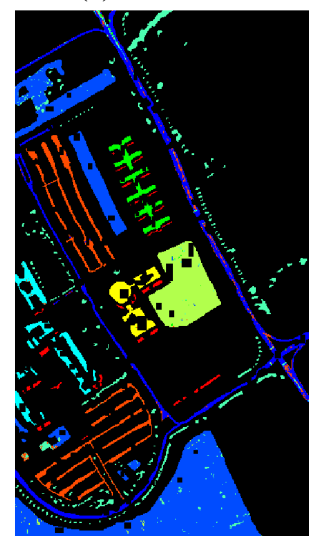

(j) $\omega-\mathrm{AP}$

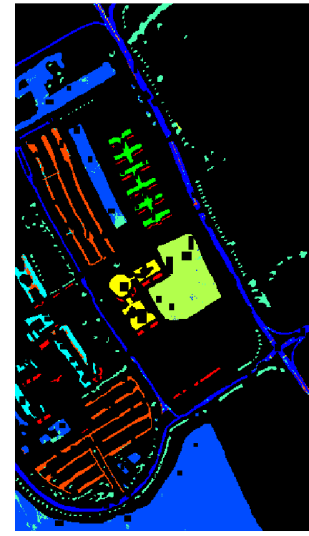

(o) $\mathrm{FP}_{\mu}$

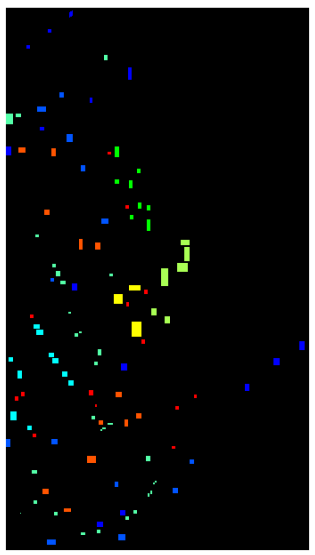

(b) Training set

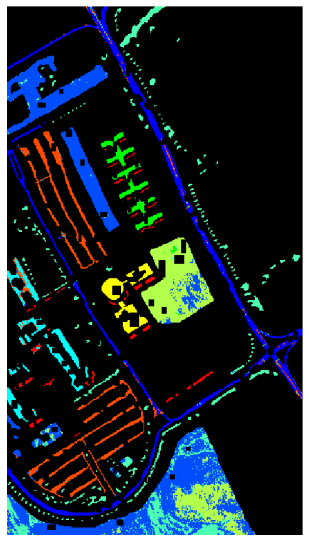

(f) AP-minT

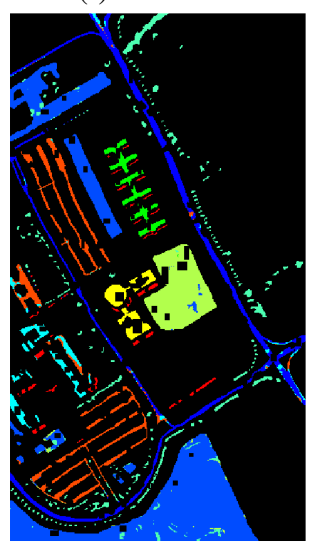

(k) LFAP

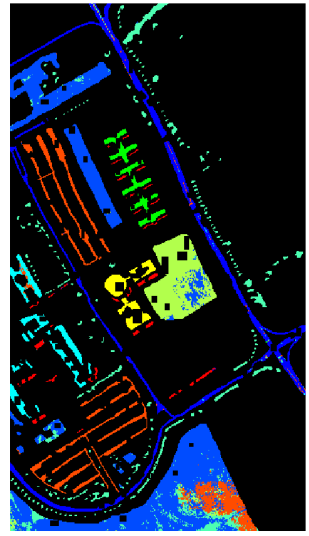

(p) $\mathrm{FP}_{a}$

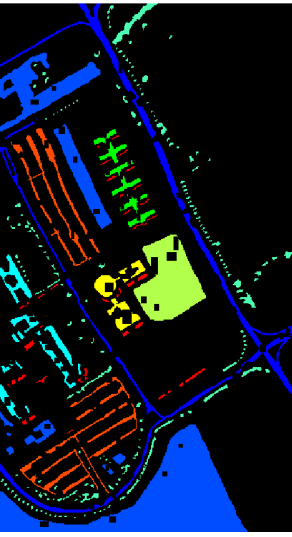

(c) Test set

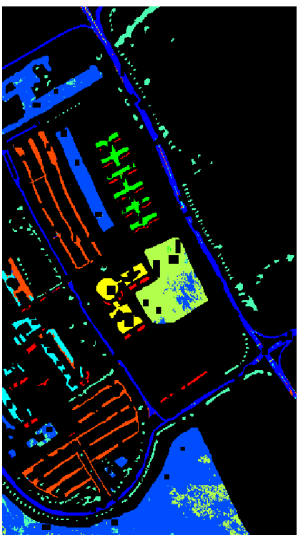

(g) AP

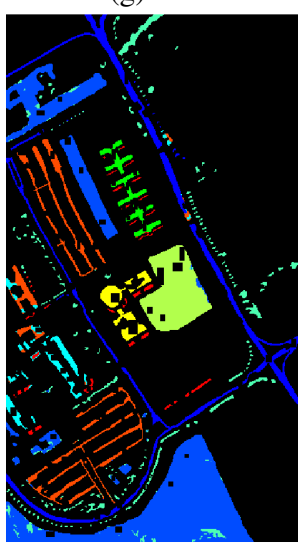

(1) LFSDAP

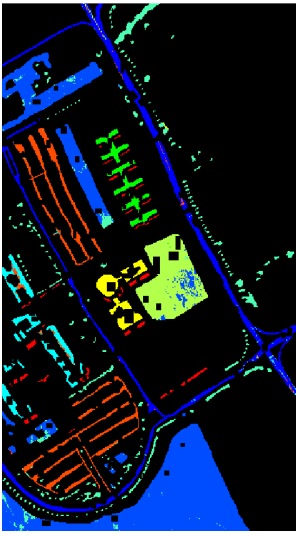

(q) $\mathrm{FP}_{\mu+a}$

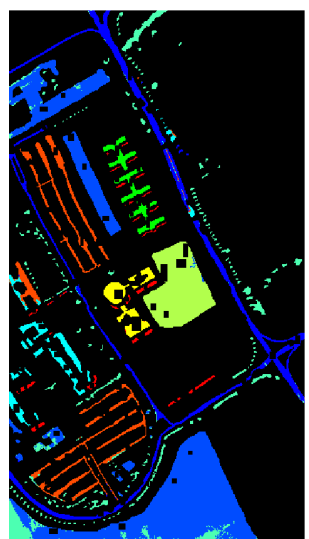

(h) SDAP

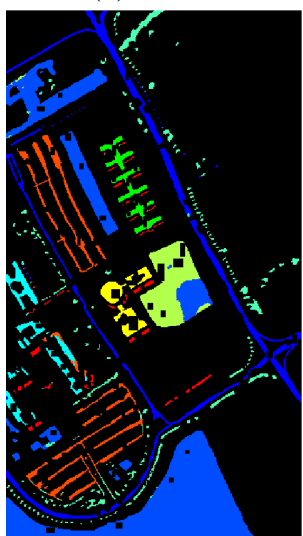

(m) HAP

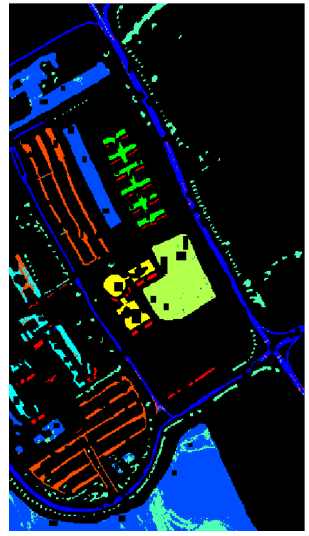

(r) TF-AP

Fig. 5: Classification results of Pavia data corresponding to the results of table $\mathrm{VI} \square$ : trees, $\square$ : gravel, $\square$ : meadows, $\square$ : asphalt, $\square$ : metal, $\square$ : bare soil, $\square$ : bitumen, $\square$ : shadows, $\square$ : bricks. 
TABLE VIII: Classification results of Gray-Potsdam dataset obtained by different methods using the default 4-connectivity and 1-byte quantization.

\begin{tabular}{|c|c|c|c|c|}
\hline \multirow{2}{*}{ Method } & \multirow{2}{*}{ Dimension } & \multicolumn{3}{|c|}{ Classification result } \\
\hline & & $\mathrm{OA}(\%)$ & AA (\%) & $\kappa \times 100$ \\
\hline Gray-values & 1 & $46.56 \pm 0.42$ & $34.30 \pm 0.02$ & $30.84 \pm 0.32$ \\
\hline AP-maxT & 20 & $63.65 \pm 0.14$ & $59.68 \pm 0.02$ & $52.65 \pm 0.15$ \\
\hline AP-minT & 20 & $58.00 \pm 0.23$ & $56.55 \pm 0.02$ & $47.13 \pm 0.21$ \\
\hline $\mathrm{AP}$ & 38 & $75.10 \pm 0.05$ & $77.56 \pm 0.02$ & $67.83 \pm 0.05$ \\
\hline SDAP & 20 & $76.19 \pm 0.08$ & $78.25 \pm 0.02$ & $69.20 \pm 0.09$ \\
\hline$\alpha$-AP & 20 & $68.32 \pm 0.07$ & $67.94 \pm 0.04$ & $59.47 \pm 0.08$ \\
\hline$\omega-\mathrm{AP}$ & 20 & $68.10 \pm 0.06$ & $67.78 \pm 0.03$ & $59.23 \pm 0.06$ \\
\hline LFAP & 76 & $78.62 \pm 0.04$ & $81.21 \pm 0.04$ & $72.25 \pm 0.05$ \\
\hline LFSDAP & 40 & $\mathbf{8 0 . 8 0} \pm \mathbf{0 . 0 3}$ & $83.44 \pm \mathbf{0 . 0 2}$ & $75.05 \pm 0.04$ \\
\hline $\mathrm{FP}_{\mu}$ & 38 & $77.14 \pm 0.05$ & $79.41 \pm 0.02$ & $70.42 \pm 0.06$ \\
\hline $\mathrm{FP}_{a}$ & 38 & $77.19 \pm 0.05$ & $79.46 \pm 0.02$ & $70.47 \pm 0.05$ \\
\hline $\mathrm{FP}_{\mu+a}$ & 76 & $77.94 \pm 0.04$ & $80.17 \pm 0.03$ & $71.43 \pm 0.05$ \\
\hline TF-AP & 18 & $72.34 \pm 0.05$ & $74.93 \pm 0.04$ & $64.33 \pm 0.06$ \\
\hline
\end{tabular}

TABLE IX: Classification results per class of Gray-Potsdam dataset obtained by different methods using the default 4connectivity and 1-byte quantization.

\begin{tabular}{|c|c|c|c|c|c|c|c|}
\hline \multirow{2}{*}{ Method } & \multirow{2}{*}{ Dimension } & \multicolumn{6}{|c|}{ Accuracy per class } \\
\hline & & Background & Trees & Cars & Buildings & Low vegetation & Impervious surfaces \\
\hline Gray-values & 1 & $10.24 \pm 1.34$ & $16.23 \pm 1.23$ & $11.45 \pm 0.22$ & $49.82 \pm 1.51$ & $70.86 \pm 1.51$ & $47.21 \pm 1.12$ \\
\hline AP-maxT & 20 & $53.82 \pm 0.19$ & $31.80 \pm 0.63$ & $59.40 \pm 0.54$ & $68.53 \pm 0.29$ & $77.42 \pm 0.58$ & $67.08 \pm 0.62$ \\
\hline AP-minT & 20 & $54.74 \pm 0.80$ & $51.14 \pm 1.22$ & $58.37 \pm 0.19$ & $61.34 \pm 0.83$ & $67.38 \pm 0.30$ & $46.32 \pm 1.28$ \\
\hline AP & 38 & $85.43 \pm 0.21$ & $63.49 \pm 0.17$ & $92.02 \pm 0.08$ & $84.15 \pm 0.16$ & $72.42 \pm 0.21$ & $67.85 \pm 0.22$ \\
\hline SDAP & 20 & $82.57 \pm 0.48$ & $64.89 \pm 0.22$ & $92.82 \pm 0.09$ & $83.93 \pm 0.14$ & $74.43 \pm 0.23$ & $70.87 \pm 0.48$ \\
\hline$\alpha-\mathrm{AP}$ & 20 & $71.70 \pm 0.14$ & $50.39 \pm 0.30$ & $75.38 \pm 0.21$ & $80.68 \pm 0.18$ & $62.65 \pm 0.13$ & $66.86 \pm 0.24$ \\
\hline$\omega-\mathrm{AP}$ & 20 & $71.07 \pm 0.14$ & $50.26 \pm 0.42$ & $75.73 \pm 0.24$ & $80.24 \pm 0.21$ & $62.62 \pm 0.17$ & $66.76 \pm 0.22$ \\
\hline LFAP & 76 & $85.18 \pm 0.11$ & $70.54 \pm 0.17$ & $96.00 \pm 0.05$ & $84.38 \pm 0.09$ & $75.87 \pm 0.09$ & $75.30 \pm 0.07$ \\
\hline LFSDAP & 40 & $\mathbf{8 7 . 9 8} \pm \mathbf{0 . 0 9}$ & $74.25 \pm \mathbf{0 . 1 2}$ & $\mathbf{9 7 . 3 0} \pm \mathbf{0 . 0 3}$ & $86.15 \pm 0.10$ & $78.00 \pm \mathbf{0 . 0 8}$ & $76.94 \pm 0.08$ \\
\hline $\mathrm{FP}_{\mu}$ & 38 & $86.55 \pm 0.22$ & $66.47 \pm 0.17$ & $93.06 \pm 0.05$ & $85.73 \pm 0.15$ & $74.66 \pm 0.17$ & $69.97 \pm 0.18$ \\
\hline $\mathrm{FP}_{a}$ & 38 & $86.96 \pm 0.22$ & $66.42 \pm 0.18$ & $92.98 \pm 0.06$ & $85.90 \pm 0.13$ & $74.70 \pm 0.19$ & $69.81 \pm 0.21$ \\
\hline $\mathrm{FP}_{\mu+a}$ & 76 & $87.39 \pm 0.16$ & $67.60 \pm 0.14$ & $93.40 \pm 0.05$ & $86.41 \pm 0.13$ & $75.47 \pm 0.19$ & $70.74 \pm 0.18$ \\
\hline TF-AP & 18 & $82.76 \pm 0.18$ & $59.09 \pm 0.16$ & $90.47 \pm 0.10$ & $81.70 \pm 0.14$ & $69.24 \pm 0.14$ & $66.32 \pm 0.24$ \\
\hline
\end{tabular}

plementary information and eventually higher performances.

\section{Assessment of connectivity and quantization}

In this section, we discuss the influence of the connectivity parameters (4 versus 8 ) and of the quantization parameters (64 bits, 16 bits and 8 bits) in the classification results obtained with different methods.

As previously stated, the connectivity parameter is rarely mentioned in published works, though it can have a nonnegligible impact in the construction of tree representations. In general, trees computed with 4-connectivity are "finer" than the ones obtained with 8-connectivity. In other words, given the trees $T_{4}$ and $T_{8}$ obtained from the same image using 4- and 8-connectivity, respectively, every node of $T_{4}$ is a subset of a node of $T_{8}$. For instance, in Fig. 7(b) and (c), we show the max-trees of the image of Fig. 7. a) computed with 4- and 8-connectivity, respectively. It can be verified that every node of Max- $\mathrm{T}_{4}$ is a subset of a node of Max$\mathrm{T}_{8}$. As another example, the max-tree of the 4-connected Gray-Potsdam image is composed of approximately $31 \%$ more nodes than the max-tree of the 8-connected image $(4,725,207$ vs 3,606, 550 nodes). Concerning the max-trees computed on the first principal component of Pavia, the relative difference in the number of nodes is even higher: using 4-connectivity lead to approximately $43.7 \%$ more nodes than using 8 -connectivity (25, 030 vs 17, 413 nodes). Those observations raise the ques- tion of whether the connectivity has as much of an impact on the APs as it has on the number of tree nodes.

Similarly to the connectivity, the quantization parameter affects heavily the trees' depth and number of nodes. For the Pavia dataset 10 , the value of a pixel at every band is represented as a 16-bit unsigned integer. However, as aforementioned, the APs are computed on the four principal components of the Pavia dataset. Those components are obtained with the PCA.fit() method of the scikit-learn Python library, which returns real-valued (64-bit float) components. In general, rounding those real values to 16 or 8 -bit integers reduce the number of distinct values in the components and, consequently, the time and space complexity to compute their respective trees. Though the computation time and space complexity is not critical for the small sized Pavia dataset, it is of great importance for larger datasets. Hence, we will study the effect of approximating the 64-bit values to 16-bit and to 8-bit values in the classification of Pavia.

Since the post-processing techniques LFAP and HAP do not depend directly on the connectivity and quantization parameters, they will not be included in this set of experiments. Moreover, as the tree-of-shapes is not yet implemented with 8 -connectivity in the Higra package, it will not be considered in this section.

${ }^{10}$ downloaded from http://www.ehu.eus/ccwintco/index.php/Hyperspectral _Remote_Sensing_Scenes 


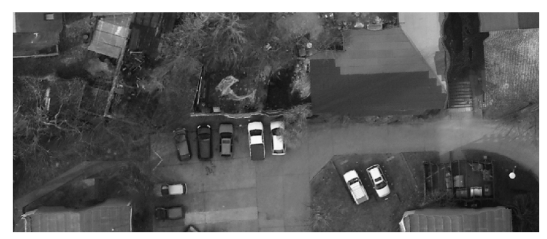

(a) Crop of Gray-Potsdam

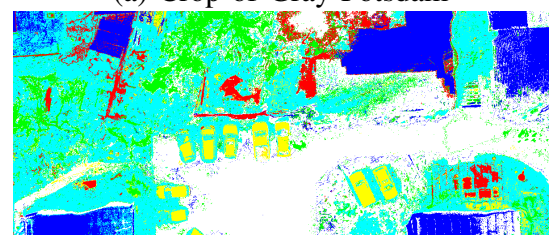

(d) AP-maxT

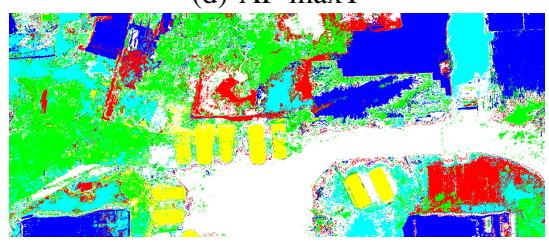

(g) SDAP

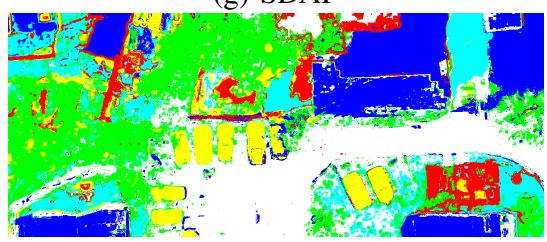

(j) LFAP

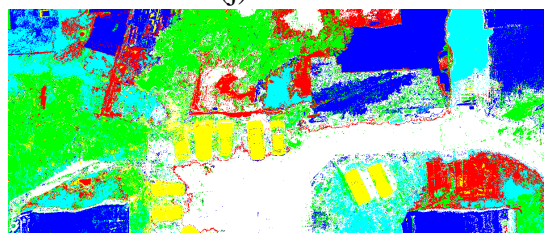

(m) $\mathrm{FP}_{a}$

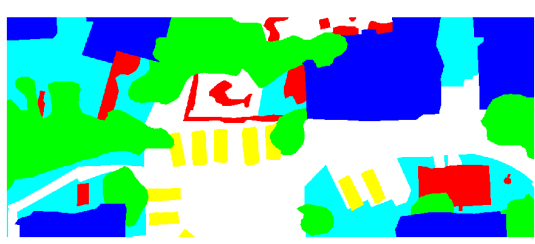

(b) Crop of ground-truth

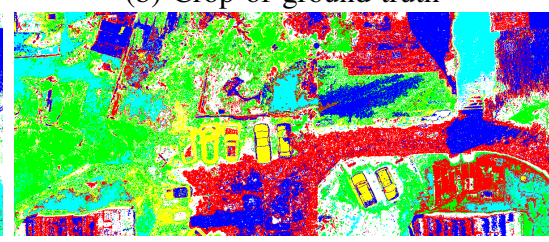

(e) AP-minT

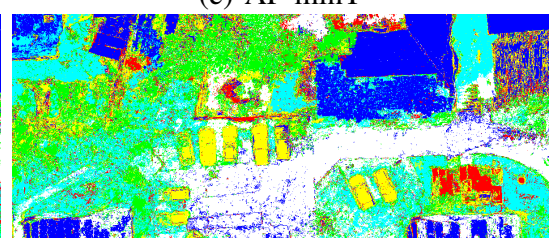

(h) $\alpha$-AP

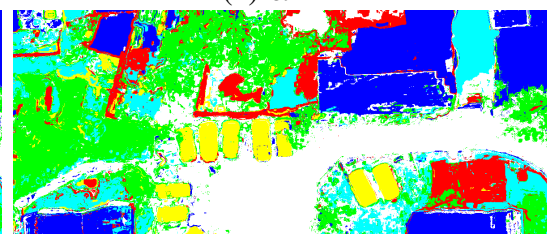

(k) LFSDAP

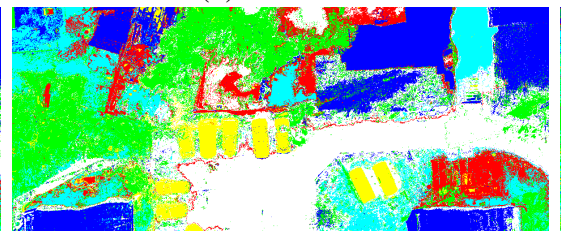

(n) $\mathrm{FP}_{\mu+a}$

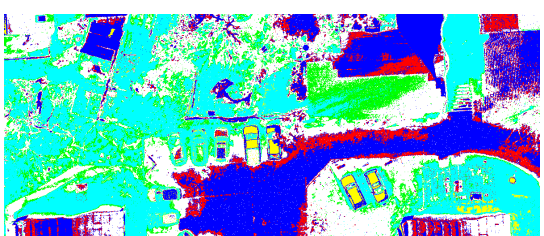

(c) Gray-scale

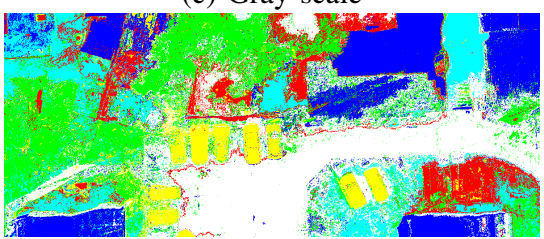

(f) AP

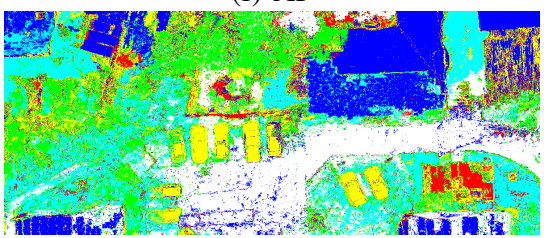

(i) $\omega$-AP

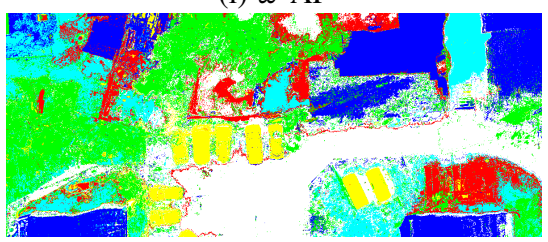

(1) $\mathrm{FP}_{\mu}$

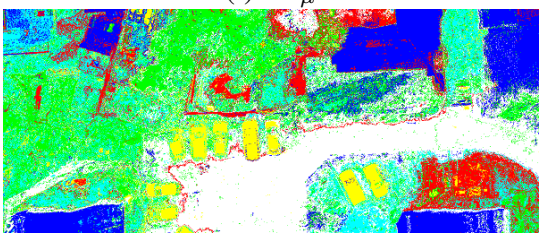

(o) TF-AP

Fig. 6: Classification results of a crop of Gray-Potsdam corresponding to the results of Table VIII $\square$ : impervious surfaces, $\square$ : buildings, $\square$ : low vegetation, $\square$ : trees, $\square$ : cars, $\square$ : background.

In Tables $\mathrm{X}$ and $\mathrm{XI}$, we present the classification results of Gray-Potsdam and Pavia data, respectively, with 4- and 8connectivity.

For the Potsdam data, the connectivity parameter had little but consistent influence on the classification results with different methods. The experiments with 4-connectivity provided better scores in general. We attribute this result to the larger number of tree nodes obtained using 4- instead of 8-connectivity. Hence, we conclude that the finer regions obtained with 4-connectivity provide valuable attributes for performing classification on this dataset.

Regarding the Pavia data, changing the connectivity parameter had a different impact on each method. All methods perform better on the 4-connected Pavia data, except for $F P_{a}$. Notably, changing the connectivity parameter of the AP-minT from 8 to 4 lead to an improvement of $8.89 \%$ and $11.02 \%$ in terms of $O A$ and $\kappa$, respectively. On the other hand, changing the connectivity parameter of the $F P_{a}$ from 4 to 8 lead to an improvement of $4.09 \%$ and $4.72 \%$ in terms of $O A$ and $\kappa$, respectively.
Overall though, 4-connectivity can be observed to outperform almost consistently 8-connectivity. In terms of future research directions, advanced connectivity concepts such as hyper-connectivity [104] and mask based connectivity [105] appear as promising options.

We now focus on the assessment of the quantization parameter in the classification of the Pavia dataset. Since the RGB values of the Potsdam dataset are already provided as 8-bit values, the quantization parameter will not be assessed on Gray-Potsdam.

In table XII we present the classification results of the Pavia dataset with 8-bit (default), 16-bit and 64-bit quantization. Regarding the classification based solely on the four principal components, approximating the 64-bit float values to 16-bit values had little influence on the results. Whereas, approximating the 64-bit/16-bit to 8-bit values had a larger impact on the classification results, with a decrease of more than $3 \%$ in terms of accuracy and $\kappa$ scores. A more significant loss is observed in the classification results with AP-minT and AP, with a decrease of more than $5 \%$ in terms of accuracy and 
TABLE X: Comparison between the classification results of Gray-Potsdam for different connectivity parameters.

\begin{tabular}{l|c|ccc|ccc}
\hline \hline \multirow{2}{*}{ Method } & \multirow{2}{*}{ Dimension } & \multicolumn{2}{|c}{ Classification result (4-connectivity) } & \multicolumn{2}{c}{ Classification result (8-connectivity) } \\
\cline { 3 - 8 } & & OA (\%) & AA (\%) & $\kappa \times 100$ & OA (\%) & AA (\%) & $\kappa \times 100$ \\
\hline AP-maxT & 20 & $\mathbf{6 3 . 6 5} \pm \mathbf{0 . 1 4}$ & $\mathbf{5 9 . 6 8} \pm \mathbf{0 . 0 2}$ & $\mathbf{5 2 . 6 5} \pm \mathbf{0 . 1 5}$ & $63.03 \pm 0.16$ & $58.90 \pm 0.02$ & $51.84 \pm 0.17$ \\
$\mathrm{AP}-\mathrm{minT}$ & 20 & $\mathbf{5 8 . 0 0} \pm \mathbf{0 . 2 3}$ & $\mathbf{5 6 . 5 5} \pm \mathbf{0 . 0 2}$ & $\mathbf{4 7 . 1 3} \pm \mathbf{0 . 2 1}$ & $57.36 \pm 0.23$ & $55.75 \pm 0.03$ & $46.30 \pm 0.21$ \\
$\mathrm{AP}$ & 38 & $\mathbf{7 5 . 1 0} \pm \mathbf{0 . 0 5}$ & $\mathbf{7 7 . 5 6} \pm \mathbf{0 . 0 2}$ & $\mathbf{6 7 . 8 3} \pm \mathbf{0 . 0 5}$ & $73.91 \pm 0.07$ & $76.42 \pm 0.03$ & $66.36 \pm 0.07$ \\
\hline$\alpha-\mathrm{AP}$ & 20 & $\mathbf{6 8 . 3 2} \pm \mathbf{0 . 0 7}$ & $\mathbf{6 7 . 9 4} \pm \mathbf{0 . 0 4}$ & $\mathbf{5 9 . 4 7} \pm \mathbf{0 . 0 8}$ & $67.67 \pm 0.08$ & $66.72 \pm 0.04$ & $58.64 \pm 0.08$ \\
$\omega-\mathrm{AP}$ & 20 & $\mathbf{6 8 . 1 0} \pm \mathbf{0 . 0 6}$ & $\mathbf{6 7 . 7 8} \pm \mathbf{0 . 0 3}$ & $\mathbf{5 9 . 2 3} \pm \mathbf{0 . 0 6}$ & $67.40 \pm 0.08$ & $66.46 \pm 0.03$ & $58.34 \pm 0.08$ \\
\hline $\mathrm{FP}_{\mu}$ & 38 & $\mathbf{7 7 . 1 4} \pm \mathbf{0 . 0 5}$ & $\mathbf{7 9 . 4 1} \pm \mathbf{0 . 0 2}$ & $\mathbf{7 0 . 4 2} \pm \mathbf{0 . 0 6}$ & $75.95 \pm 0.07$ & $78.23 \pm 0.02$ & $68.91 \pm 0.08$ \\
$\mathrm{FP}_{a}$ & 38 & $\mathbf{7 7 . 1 9} \pm \mathbf{0 . 0 5}$ & $\mathbf{7 9 . 4 6} \pm \mathbf{0 . 0 2}$ & $\mathbf{7 0 . 4 7} \pm \mathbf{0 . 0 5}$ & $75.98 \pm 0.09$ & $78.28 \pm 0.03$ & $68.96 \pm 0.10$ \\
$\mathrm{FP}_{\mu+a}$ & 76 & $\mathbf{7 7 . 9 4} \pm \mathbf{0 . 0 4}$ & $\mathbf{8 0 . 1 7} \pm \mathbf{0 . 0 3}$ & $\mathbf{7 1 . 4 3} \pm \mathbf{0 . 0 5}$ & $76.70 \pm 0.06$ & $78.96 \pm 0.03$ & $69.86 \pm 0.07$ \\
$\mathrm{TF}-\mathrm{AP}$ & 18 & $\mathbf{7 2 . 3 4} \pm \mathbf{0 . 0 5}$ & $\mathbf{7 4 . 9 3} \pm \mathbf{0 . 0 4}$ & $\mathbf{6 4 . 3 3} \pm \mathbf{0 . 0 6}$ & $71.90 \pm 0.08$ & $74.49 \pm 0.03$ & $63.81 \pm 0.09$ \\
\hline
\end{tabular}

TABLE XI: Comparison between the classification results of Pavia for different connectivity parameters.

\begin{tabular}{|c|c|c|c|c|c|c|c|}
\hline \multirow{2}{*}{ Method } & \multirow{2}{*}{ Dimension } & \multicolumn{3}{|c|}{ Classification result (4-connectivity) } & \multicolumn{3}{|c|}{ Classification result (8-connectivity) } \\
\hline & & $\mathrm{OA}(\%)$ & AA (\%) & $\kappa \times 100$ & $\mathrm{OA}(\%)$ & AA $(\%)$ & $\kappa \times 100$ \\
\hline AP-maxT & 80 & $89.21 \pm 0.63$ & $87.37 \pm 0.19$ & $85.42 \pm 0.80$ & $87.88 \pm 1.16$ & $89.56 \pm 0.30$ & $84.17 \pm 1.43$ \\
\hline AP-minT & 80 & $87.11 \pm 2.00$ & $92.62 \pm 0.61$ & $83.23 \pm 2.41$ & $78.22 \pm 0.50$ & $88.26 \pm 0.14$ & $72.21 \pm 0.57$ \\
\hline AP & 152 & $90.57 \pm 2.60$ & $93.13 \pm 0.67$ & $\mathbf{8 7 . 5 3} \pm \mathbf{3 . 3 2}$ & $90.54 \pm 1.55$ & $93.80 \pm 0.40$ & $87.31 \pm 1.99$ \\
\hline$\alpha-\mathrm{AP}$ & 80 & $95.46 \pm 0.59$ & $95.25 \pm 1.23$ & $93.91 \pm 0.79$ & $94.73 \pm 0.40$ & $95.66 \pm 0.27$ & $92.97 \pm 0.52$ \\
\hline$\omega-\mathrm{AP}$ & 80 & $96.33 \pm 0.40$ & $\mathbf{9 7 . 1 7} \pm \mathbf{0 . 8 9}$ & $95.08 \pm 0.54$ & $94.71 \pm 0.34$ & $95.69 \pm 0.17$ & $92.94 \pm 0.44$ \\
\hline $\mathrm{FP}_{\mu}$ & 152 & $96.76 \pm 0.15$ & $\mathbf{9 7 . 0 5} \pm \mathbf{0 . 1 9}$ & $95.65 \pm 0.21$ & $93.35 \pm 0.15$ & $93.55 \pm 0.16$ & $90.90 \pm 0.20$ \\
\hline $\mathrm{FP}_{a}$ & 152 & $85.48 \pm 0.98$ & $93.94 \pm 0.55$ & $81.36 \pm 1.16$ & $89.57 \pm 1.18$ & $94.03 \pm 0.32$ & $86.08 \pm 1.50$ \\
\hline $\mathrm{FP}_{\mu+a}$ & 304 & $96.15 \pm 0.14$ & $96.74 \pm 0.32$ & $94.82 \pm 0.19$ & $94.21 \pm 0.32$ & $95.00 \pm 0.28$ & $92.09 \pm 0.44$ \\
\hline TF-AP & 72 & $\mathbf{9 3 . 9 8} \pm \mathbf{0 . 5 3}$ & $96.26 \pm 0.29$ & $92.04 \pm 0.68$ & $92.65 \pm 0.24$ & $95.26 \pm 0.17$ & $90.32 \pm 0.31$ \\
\hline
\end{tabular}

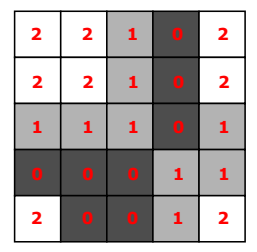

(a) Image

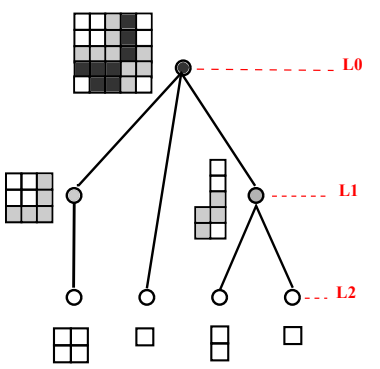

(b) $\mathrm{Max}-\mathrm{T}_{4}$

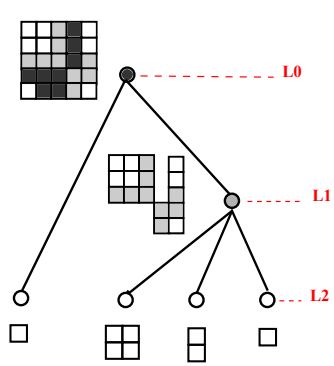

(c) $\mathrm{Max}-\mathrm{T}_{8}$
Fig. 7: (a) Original grayscale image $X: E \rightarrow[0,1,2]$. (b) Max-tree of $X$ computed using 4-connectivity. (c) Max-tree of $X$ computed using 8-connectivity.

$\kappa$ scores when the 64-bit float values are approximated to 8-bit values. On the other hand, we have opposite results for SDAP, $\alpha$-AP and $\omega$-AP: using 8-bit for quantization provides the best scores. Consequently, we could draw different conclusions regarding the performance of APs compared to SDAPs, $\alpha$ $\mathrm{AP}$ and $\omega$-AP depending on the quantization settings: if 8-bit, then SDAP, $\alpha$-AP and $\omega$-AP outperform AP; otherwise, AP outperform those three methods. Regarding feature profiles, the best $F P_{\mu}$ and $F P_{\mu+a}$ results are achieved with 64-bit quantization. Whereas, the best $F P_{a}$ is achieved with 8-bit quantization. It is noteworthy that, as discussed previously, $F P_{\mu}$ and $F P_{a}$ also presented opposite results with respect to the connectivity parameters. This may suggest that having finer (resp. coarser) components trees, obtained with 4-connectivity and 64-bit quantization (resp. 8-connectivity and 8-bit quantization) for example, lead to better $F P_{\mu}$ (resp. $F P_{a}$ ) scores.

Our experiments show that besides the choice of attributes and threshold values, the connectivity and quantization parameters have a great impact as well on the performance of APs with different tree representations. More importantly, this effect varies greatly depending on the underlying tree type. In summary, these two parameters that are almost always silently set to (unmentioned in published studies) default values, appear to merit the same level of attention and care that threshold/attribute selection enjoys.

\section{DisCUSSION ON THE GENERALIZATION OF APS}

The experiments described in the previous section follow the same approach of other experiments in the literature: the training and testing features are obtained from the same tree computed on the whole data (or on the principal components of the data). This approach is reasonable when the aim is to completely classify the pixels of an image whose annotated pixels are evenly spread across this image [12]. That was the case of the training sets considered previously, which allowed us to obtain an improvement of more than $30 \%$ in terms of classification accuracy using APs with respect to spectral pixel values. However, this technique raises doubts in case of situations where one encounters distinct images for training and testing, or alternatively when the training pixels are not evenly spread across the data. This issue has already been discussed in [17], where the authors show that having training samples evenly spread across all ground-truth connected components is not a realistic scenario for evaluating classification methods in the remote sensing context.

In this section, we discuss the generalization of APs in those two scenarios. We first address in Sec. $\mathrm{V}$-A the problem of 
TABLE XII: Comparison between the classification results of Pavia for different quantization parameters.

\begin{tabular}{|c|c|c|c|c|c|c|c|c|c|c|}
\hline \multirow{2}{*}{ Method } & \multirow{2}{*}{ Dimension } & \multicolumn{3}{|c|}{ Classification result (8-bit) } & \multicolumn{3}{|c|}{ Classification result (16-bit) } & \multicolumn{3}{|c|}{ Classification result (64-bit) } \\
\hline & & OA (\%) & AA (\%) & $\kappa \times 100$ & OA (\%) & AA (\%) & $\kappa \times 100$ & OA (\%) & AA (\%) & $\kappa \times 100$ \\
\hline $4 \mathrm{PC}$ & 4 & $65.27 \pm 0.25$ & $74.88 \pm 0.20$ & $56.93 \pm 0.27$ & $68.60 \pm \mathbf{0 . 1 0}$ & $78.08 \pm \mathbf{0 . 0 9}$ & $60.93 \pm 0.11$ & $68.51 \pm 0.24$ & $78.08 \pm \mathbf{0 . 2 2}$ & $60.81 \pm 0.29$ \\
\hline AP-maxT & 80 & $89.21 \pm 0.63$ & $87.37 \pm 0.19$ & $85.42 \pm 0.80$ & $88.97 \pm 0.20$ & $87.39 \pm 0.15$ & $84.99 \pm 0.27$ & $89.07 \pm 0.37$ & $87.42 \pm 0.17$ & $85.13 \pm 0.49$ \\
\hline AP-minT & 80 & $87.11 \pm 2.00$ & $92.62 \pm 0.61$ & $83.23 \pm 2.41$ & $91.58 \pm 1.64$ & $94.55 \pm 0.65$ & $88.89 \pm 2.06$ & $92.42 \pm 1.07$ & $94.77 \pm 0.83$ & $89.97 \pm \mathbf{1 . 3 5}$ \\
\hline $\mathrm{AP}$ & 152 & $90.57 \pm 2.60$ & $93.13 \pm 0.67$ & $87.53 \pm 3.32$ & $95.43 \pm 0.21$ & $94.01 \pm 0.47$ & $93.83 \pm 0.28$ & $95.50 \pm 0.19$ & $94.25 \pm 0.52$ & $93.93 \pm 0.26$ \\
\hline SDA & 80 & $94.10 \pm 0.21$ & $\mathbf{9 3 . 8 5} \pm \mathbf{0 . 3 7}$ & $92.16 \pm 0.27$ & $92.29 \pm 0.32$ & $91.36 \pm 0.42$ & $89.66 \pm 0.42$ & $92.49 \pm 0.47$ & $91.34 \pm 0.61$ & $89.92 \pm 0.61$ \\
\hline$\alpha-\mathrm{AP}$ & 80 & $95.46 \pm 0.59$ & $95.25 \pm 1.23$ & $93.91 \pm 0.79$ & $93.87 \pm 0.72$ & 93.85 & $91.82 \pm 0.93$ & $94.08 \pm 0.17$ & 93.96 & $92.10 \pm 0.22$ \\
\hline$\omega$-AP & 80 & $96.33 \pm 0.40$ & $\mathbf{9 7 . 1 7} \pm \mathbf{0 . 8 9}$ & $95.08 \pm 0.54$ & $93.75 \pm 0.54$ & $92.97 \pm 0.76$ & $91.64 \pm 0.71$ & $93.75 \pm 0.44$ & $92.78 \pm 0.98$ & $91.64 \pm 0.59$ \\
\hline $\mathrm{FP}_{\mu}$ & 152 & $96.76 \pm 0.15$ & $97.05 \pm 0.19$ & $95.65 \pm 0.21$ & $97.09 \pm 0.70$ & $97.43 \pm 0.47$ & $96.10 \pm 0.93$ & $97.10 \pm 1.00$ & $97.64 \pm 0.33$ & $96.11 \pm 1.31$ \\
\hline $\mathrm{FP}_{a}$ & 152 & $\mathbf{8 5 . 4 8} \pm \mathbf{0 . 9 8}$ & $\mathbf{9 3 . 9 4} \pm \mathbf{0 . 5 5}$ & $81.36 \pm 1.16$ & $82.72 \pm 0.74$ & $92.64 \pm 0.63$ & $77.99 \pm 0.95$ & $83.09 \pm 0.42$ & $93.05 \pm 0.29$ & $78.50 \pm 0.55$ \\
\hline $\mathrm{FP}_{\mu+a}$ & 304 & $96.15 \pm 0.14$ & $96.74 \pm 0.32$ & $94.82 \pm 0.19$ & $97.48 \pm 0.31$ & $98.30 \pm 0.19$ & $96.62 \pm 0.41$ & $\mathbf{9 7 . 5 5} \pm \mathbf{0 . 2 9}$ & $98.23 \pm 0.26$ & $96.71 \pm 0.39$ \\
\hline
\end{tabular}

having data composed of a single image, but with a better separation between training and testing pixels. To do so, we perform experiments using a new split of the Gray-Potsdam and Pavia datasets. Then, in Sec. V-B, we approach the case where training and testing sets belong to different images. We split the Gray-Potsdam image so that APs can be computed separately for training and testing pixels.

\section{A. Generalization of APs to other partially annotated images}

The "standard" training set of the Pavia University dataset, i.e. the training set of Pavia 1 , is composed of pixels belonging to most of the ground-truth (and testing) connected components. Hence, the training set of each semantic class accounts for most of the variability in terms of spectral signatures and geometric properties of the connected components of the said class. However, the availability of training samples from every connected component of each semantic class in a real-world scenario, dealing commonly with remote sensing datasets representing geographically large areas, is evidently unrealistic. The data splitting procedure used in this section aims to simulate, at a limited degree, the aforementioned realworld conditions.

We compare the classification results presented in the previous section with the results based on a new partition of the Pavia and Gray-Potsdam datasets ${ }^{11}$. In the new partition of Pavia (see Fig. 9(b) and (c)), ten sets of training samples were randomly extracted from a restricted region (composed of 85 connected components) of the ground-truth, which is composed of 229 connected components in total. In order to provide comparable results with the standard partition of this dataset, we selected the same number of training samples per class, resulting in 3921 training samples (see Table I) and 40,002 testing samples for each of the ten random splits. Similarly, ten sets of training samples of Gray-Potsdam were extracted from nearly half of the ground-truth connected components. In total, 726 ground-truth connected components (see Fig. 8) contributed to the new training sets of GrayPotsdam. The same number of training and test samples given in Table II were obtained. These new partitions of the Pavia and Gray-Potsdam datasets will be denoted as Pavia ${ }_{2}$ and GrayPotsdam $_{2}$, respectively. Both training and testing sets include pixels belonging to all classes of those datasets. The experiments were performed using the default settings given

${ }^{11}$ The proposed split of Pavia and Gray-Potsdam datasets are available in https://gitlab.inria.fr/dsantana/attributes-profiles-survey-source-codes

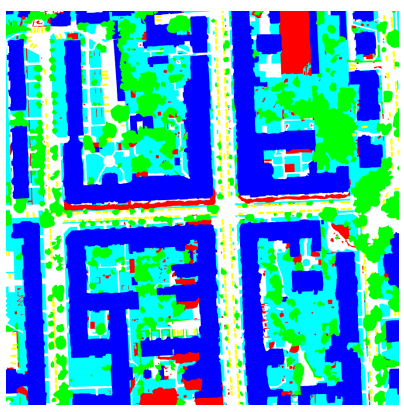

(a)

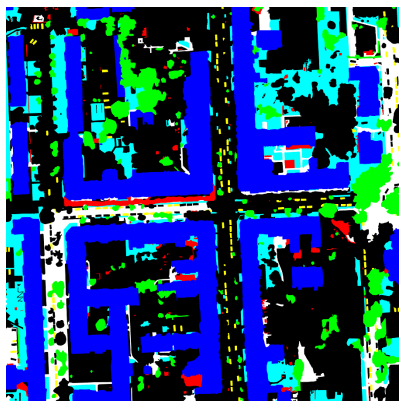

(b)
Fig. 8: (a) Original Gray-Potsdam ground-truth. (b) Connected components that contributed to the training set of the partition GrayPotsdam $_{2}$.

in Sec. IV; 4-connectivity and 8-bit quantization. We applied the same set of thresholds for the area and moment of inertia attributes used in the previous experiments on Gray-Potsdam and Pavia.

The main challenge of using those new dataset partitions is that a sample of a given semantic class is not always representative of other samples of the same class in different regions the image. This is particularly true for the bare soil and meadows classes of Pavia. In the classification results based only on pixel intensities, several meadow samples are classified as bare soil and vice-versa, as shown in Figure 5 (d). A similar observation can be drawn for the building and impervious surface classes of Gray-Potsdam (see Figure 6. (c)).

Tables XIII and XIV present the classification results computed on the partitions Pavia (Fig. 3) and Pavia $_{2}$ (Fig. 9]. First, in Table XIII, we see a degradation in the baseline classification results (4 PC) using the new partition, which is due to the training set not including diversified samples of every class. Then, we can observe an even larger degradation in terms of AP results. With Pavia 1 , all APs improve the baseline results by $20.21-31.49 \%$ in terms of overall accuracy while that, with $\mathrm{Pavia}_{2}$, the improvement ranges from 1.65 to $15.99 \%$. Moreover, with Pavia $_{2}$, as shown in Tab. XIV none of the AP-based methods outperform the baseline with respect to the brick class, and we observe a less remarkable improvement for the meadow class. On the other hand, the best scores achieved with Pavia $_{2}$ for the other seven classes are comparable with the best scores achieved with Pavia $a_{1}$. For the Pavia dataset, we can conclude that the classification 
results with AP-based methods presented in Sec. IV cannot be generalized to more realistic scenarios (with better separation between training and test pixels). In fact, it appears that APs are much less useful when the training and test samples belong to regions with dissimilar spatial and geometric properties. For instance, the training and test pixels of the asphalt class all belong to thin and elongated regions, which may be the reason why the results for this class are improved by all AP-based methods with respect to the baseline. On the other hand, the pixels in the training and test sets of the meadow class belong to components of very different shapes, which may partially explain the worse results on this class.

Fig. 9(d)-(n) illustrates the classification results of Table XIII We can observe that all AP-based methods improve considerably the classification results of the bare soil class (in light green) with respect to the baseline results (Fig. 9(d)). This can be due to the training and test pixels sharing nodes/features at higher levels of the trees. On the other hand, the classification results of the shadow and meadow classes are worsened by at least half of those methods.

We now compare the results between the partitions GrayPotsdam $_{1}$ and GrayPotsdam 2 of Gray-Potsdam dataset. Tab. XV presents the classification results obtained

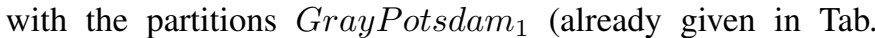
VIII) and GrayPotsdam 2 . Similarly to Pavia, we can observe a significant drop in classification accuracy for all AP-based methods when the new partition GrayPotsdam 2 is used. While APs improve the baseline scores by up to $31.38 \%$ on the GrayPotsdam 1 partition, the classification improvements do not exceed $10.55 \%$ on the new partition GrayPotsdam 2 . Moreover, the classification results of two of the six classes (low vegetation and impervious surfaces) are worsened by most of the AP-based methods, as shown in Tab. XVI

Fig. 10(d)-(n) illustrate the classification results on the crop of Gray-Potsdam given in Fig. 10(b) (same as Fig. 6(b)) obtained with the partition GrayPotsdam ${ }_{2}$. The regions of the cropped image which contributed to the training set of GrayPotsdam $_{2}$ are shown in Fig. 10(c). Our main observation is that the classification results of the regions that do not contribute to the training sets are much poorer if compared to other regions of the same class. For instance, among the pixels belonging to the largest connected component of the the impervious class (which does not contribute to the training sets), virtually no pixels are correctly classified by the tested methods. In contrast, the pixels of this region are fairly well classified when using the partition GrayPotsdam 1 , as shown in Fig. 6

Overall, using the new partitions $\mathrm{Pavia}_{2}$ and GrayPotsdam $_{2}$, we observed a very significant drop of performance across all approaches. Moreover, the drop in performance occurred at different degrees for each thematic class of the datasets. This may be linked to the fact that some classes are composed of regions with similar geometric properties (e.g. the 'asphalt' class of Pavia and the 'car' class of Gray-Potsdam) while this is not true for the other classes (e.g. the 'meadow' class of Pavia and the 'low vegetation' class of Gray-Potsdam). However, a deeper investigation is necessary to confirm this assumption.

\section{B. Generalization of APs to multiple-image datasets}

Though the partition $\mathrm{Pavia}_{2}$ better separates training and test samples when compared to Pavia ${ }_{1}$, training and test pixels of $\mathrm{Pavia}_{2}$ may still share features obtained from nodes at higher levels of the tree. The same holds for the partition GrayPotsdam $_{2}$. In this section, we go one step further to completely separate the computation of training and test features.

As mentioned previously, the Potsdam data set is composed of several patches covering a large urban scene. The original ISPRS labeling contest consisted in providing the classification labels for the pixels on the testing patches based on the information given by the training patches. Hence, extracting training and testing features from the same tree is only possible if all patches are connected in the real scene. Otherwise, we would need to compute tree representations separately on the training and testing patches. To approach the generalization of APs to this kind of problem, we split the Gray-Potsdam image in a way that two independent component trees can be computed for the training and testing samples. This way, we ensure that the training and testing pixels will not share any nodes along the trees. Alternatively, we could have chosen two neighbour image patches from the Potsdam dataset, but it wouldn't contribute more to the generalization of the method than splitting Gray-Potsdam in two.

The Gray-Potsdam dataset was divided in two halves (see Fig. 11) such that ten sets of training and test samples were extracted from the upper and lower half, respectively. From the upper half, we randomly selected 60,000 pixels of each class, as done for the other partitions of this dataset. The test set is composed of all 18,000,000 pixels in the lower half of the image. This partition of Gray-Potsdam will be denoted as GrayPotsdam 3 in the remainder of this section. To evaluate the generalization of APs in this scenario, we performed experiments following two approaches: the standard one, where a single hierarchical representation is computed on the whole image and, then, used to extract training and test features; and a new approach, where two independent trees are computed on the training and test images and, hence, the training and test features are obtained from distinct trees.

Tables XVII and XVIII present the overall and perclass classification results, respectively, with the partition GrayPotsdam $_{3}$ following those two approaches.

Our first observation is that the baseline results on the partition GrayPotsdam 3 present slightly higher scores when compared to the baseline results on GrayPotsdam ${ }_{1}$ and GrayPotsdam $_{2}$. On the other hand, we observe an even larger drop in performance for all AP-based approaches with respect to the previous experiments. Following the usual approach, with a single tree computed from the whole data, six over nine methods outperform the baseline. The best method $(\omega$ AP), improves the baseline by $5.85 \%, 10.58 \%$ and $8.63 \%$ in terms of OA, AA and $\kappa$ scores, respectively. On the other hand, with two trees computed independently on the training and test images, only three over nine methods outperform the baseline to a smaller degree. The best approach $\left(F P_{\mu+a}\right)$, improves the baseline by $3.88 \%, 5.46 \%$ and $3.96 \%$ in terms of OA, AA 
TABLE XIII: Classification results of the Pavia dataset performed on the training/testing sets from standard partition Pavia 1 (Fig. 3 and new partition Pavia $_{2}$ (Fig. 9).

\begin{tabular}{|c|c|c|c|c|c|c|c|}
\hline \multirow{2}{*}{ Method } & \multirow{2}{*}{ Dimension } & \multicolumn{3}{|c|}{ Classification result $\left(\right.$ Pavia $\left._{1}\right)$} & \multicolumn{3}{|c|}{ Classification result $\left(\right.$ Pavia $\left._{2}\right)$} \\
\hline & & OA (\%) & AA (\%) & $\kappa \times 100$ & OA (\%) & AA (\%) & $\kappa \times 100$ \\
\hline $4 \mathrm{PC}$ & 4 & $65.27 \pm 0.25$ & $74.88 \pm 0.20$ & $56.93 \pm 0.27$ & $59.58 \pm 0.74$ & $73.44 \pm 0.45$ & $50.76 \pm 0.76$ \\
\hline AP-maxT & 80 & $89.21 \pm 0.63$ & $87.37 \pm 0.19$ & $85.42 \pm 0.80$ & $61.23 \pm 0.68$ & $82.27 \pm 0.36$ & $54.42 \pm 0.69$ \\
\hline AP-minT & 80 & $87.11 \pm 2.00$ & $92.62 \pm 0.61$ & $83.23 \pm 2.41$ & $68.72 \pm 2.72$ & $79.89 \pm 1.06$ & $62.03 \pm 2.88$ \\
\hline AP & 152 & $90.57 \pm 2.60$ & $93.13 \pm 0.67$ & $87.53 \pm 3.32$ & $64.49 \pm 1.35$ & $84.00 \pm 0.74$ & $57.90 \pm 1.38$ \\
\hline SDAP & 80 & $94.10 \pm 0.21$ & $93.85 \pm 0.37$ & $92.16 \pm 0.27$ & $76.63 \pm 3.70$ & $82.05 \pm 1.08$ & $70.85 \pm 4.09$ \\
\hline$\alpha-\mathrm{AP}$ & 80 & $95.46 \pm 0.59$ & $95.25 \pm 1.23$ & $93.91 \pm 0.79$ & $64.72 \pm 0.89$ & $76.02 \pm 1.19$ & $57.32 \pm 1.11$ \\
\hline$\omega-\mathrm{AP}$ & 80 & $96.33 \pm 0.40$ & $\mathbf{9 7 . 1 7} \pm \mathbf{0 . 8 9}$ & $95.08 \pm 0.54$ & $63.62 \pm 1.50$ & $74.59 \pm 0.78$ & $55.96 \pm 1.55$ \\
\hline $\mathrm{FP}_{\mu}$ & 152 & $96.76 \pm 0.15$ & $97.05 \pm 0.19$ & $95.65 \pm 0.21$ & $75.57 \pm 1.43$ & $85.36 \pm 2.14$ & $69.61 \pm 1.63$ \\
\hline $\mathrm{FP}_{a}$ & 152 & $85.48 \pm 0.98$ & $93.94 \pm 0.55$ & $81.36 \pm 1.16$ & $66.40 \pm 1.17$ & $81.48 \pm 0.39$ & $59.80 \pm 1.17$ \\
\hline $\mathrm{FP}_{\mu+a}$ & 304 & $96.15 \pm 0.14$ & $96.74 \pm 0.32$ & $94.82 \pm 0.19$ & $72.06 \pm 2.91$ & $82.83 \pm 0.83$ & $65.81 \pm 3.15$ \\
\hline TF-AP & 72 & $93.98 \pm 0.53$ & $96.26 \pm 0.29$ & $92.04 \pm 0.68$ & $69.25 \pm 5.60$ & $84.46 \pm 1.80$ & $63.22 \pm 5.94$ \\
\hline
\end{tabular}

TABLE XIV: Classification results per class of the Pavia dataset performed on the partition Pavia 2 (Fig. 9 b)-(c)).

\begin{tabular}{|c|c|c|c|c|c|c|c|c|c|c|}
\hline \multirow{2}{*}{ Method } & \multirow{2}{*}{ Dimension } & \multicolumn{9}{|c|}{ Accuracy per class (\%) } \\
\hline & & Asphalt & Meadow & Gravel & Tree & Metal & Soil & Bitumen & Brick & Shadow \\
\hline $4 \mathrm{PC}$ & 4 & $82.34 \pm 0.97$ & $41.69 \pm 1.49$ & $44.88 \pm 2.67$ & $98.09 \pm 0.29$ & $98.43 \pm 0.47$ & $59.82 \pm 1.80$ & $75.59 \pm 3.21$ & $60.84 \pm 3.01$ & $99.28 \pm 0.16$ \\
\hline AP-maxT & 80 & $95.06 \pm 1.34$ & $30.95 \pm 1.30$ & $87.22 \pm 2.68$ & $98.25 \pm 0.19$ & $99.70 \pm 0.11$ & $94.68 \pm 0.75$ & $97.43 \pm 0.42$ & $37.23 \pm 0.16$ & $99.90 \pm 0.15$ \\
\hline AP-minT & 80 & $92.49 \pm 0.80$ & $49.23 \pm 6.13$ & $86.24 \pm 8.02$ & $98.64 \pm 0.21$ & $99.33 \pm 0.39$ & $97.61 \pm 0.46$ & $99.90 \pm 0.09$ & $38.20 \pm 0.21$ & $57.41 \pm 1.14$ \\
\hline $\mathrm{AP}$ & 152 & $97.50 \pm 1.34$ & $35.74 \pm 2.82$ & $92.41 \pm 4.09$ & $98.85 \pm 0.17$ & $99.69 \pm 0.11$ & $98.34 \pm 0.87$ & $100.0 \pm 0.00$ & $37.45 \pm 0.45$ & $96.00 \pm 6.05$ \\
\hline SDAP & 80 & $98.34 \pm 0.44$ & $64.60 \pm 8.24$ & $89.62 \pm 6.95$ & $96.04 \pm 0.39$ & $99.44 \pm 0.20$ & $99.34 \pm 0.19$ & $99.71 \pm 0.34$ & $37.16 \pm 0.03$ & $54.16 \pm 0.13$ \\
\hline$\alpha-\mathrm{AP}$ & 80 & $95.71 \pm 0.55$ & $43.02 \pm 0.47$ & $32.89 \pm 0.04$ & $99.10 \pm 0.18$ & $99.10 \pm 0.25$ & $98.56 \pm 0.16$ & $99.50 \pm 0.03$ & $41.13 \pm 10.79$ & $75.18 \pm 1.71$ \\
\hline$\omega$-AP & 80 & $95.65 \pm 0.41$ & $41.58 \pm 3.25$ & $32.89 \pm 0.07$ & $99.06 \pm 0.19$ & $99.15 \pm 0.28$ & $98.39 \pm 0.30$ & $99.51 \pm 0.06$ & $38.09 \pm 1.46$ & $67.01 \pm 7.05$ \\
\hline $\mathrm{FP}_{\mu}$ & 152 & $96.86 \pm 0.93$ & $60.86 \pm 2.52$ & $98.62 \pm 1.14$ & $98.94 \pm 0.15$ & $99.70 \pm 0.13$ & $96.41 \pm 0.88$ & $100.0 \pm 0.00$ & $38.17 \pm 2.37$ & $78.65 \pm 17.0$ \\
\hline $\mathrm{FP}_{a}$ & 152 & $95.33 \pm 1.21$ & $40.76 \pm 2.82$ & $99.53 \pm 0.49$ & $98.85 \pm 0.20$ & $99.68 \pm 0.17$ & $98.88 \pm 0.35$ & $100.0 \pm \mathbf{0 . 0 0}$ & $41.30 \pm 4.53$ & $59.01 \pm 1.31$ \\
\hline $\mathrm{FP}_{\mu+a}$ & 304 & $96.32 \pm 1.34$ & $53.13 \pm 6.58$ & $99.84 \pm 0.09$ & $98.88 \pm 0.16$ & $99.73 \pm 0.11$ & $98.28 \pm 0.40$ & $100.0 \pm 0.00$ & $40.70 \pm 4.47$ & $58.63 \pm 1.76$ \\
\hline TF-AP & 72 & $95.72 \pm 0.59$ & $45.59 \pm 11.73$ & $62.79 \pm 6.58$ & $98.99 \pm 0.15$ & $99.69 \pm 0.12$ & $98.72 \pm 0.27$ & $100.0 \pm \mathbf{0 . 0 0}$ & $58.70 \pm 9.10$ & $\mathbf{9 9 . 9 5} \pm \mathbf{0 . 0 8}$ \\
\hline
\end{tabular}

TABLE XV: Classification results obtained with the partitions GrayPotsdam 1 and GrayPotsdam G $_{2}$ the Gray-potsdam dataset.

\begin{tabular}{l|c|ccc|ccc}
\hline \hline \multirow{2}{*}{ Method } & \multirow{2}{*}{ Dimension } & \multicolumn{2}{|c}{ Classification result $\left.(\text { GrayPotsdam })_{1}\right)$} & \multicolumn{2}{c}{ Classification result (GrayPotsdam $\left.m_{2}\right)$} \\
\cline { 3 - 8 } & & OA (\%) & AA (\%) & $\kappa \times 100$ & OA (\%) & AA (\%) & $\kappa \times 100$ \\
\hline Gray-values & 1 & $46.56 \pm 0.42$ & $34.30 \pm 0.02$ & $30.84 \pm 0.32$ & $47.76 \pm 0.29$ & $34.34 \pm 0.04$ & $32.15 \pm 0.21$ \\
AP-maxT & 20 & $63.65 \pm 0.14$ & $59.68 \pm 0.02$ & $52.65 \pm 0.15$ & $52.80 \pm 0.19$ & $45.39 \pm 0.09$ & $39.13 \pm 0.16$ \\
AP-minT & 20 & $58.00 \pm 0.23$ & $56.55 \pm 0.02$ & $47.13 \pm 0.21$ & $46.58 \pm 0.16$ & $44.32 \pm 0.09$ & $32.97 \pm 0.14$ \\
AP & 38 & $75.10 \pm 0.05$ & $77.56 \pm 0.02$ & $67.83 \pm 0.05$ & $57.11 \pm 0.19$ & $55.54 \pm 0.12$ & $44.50 \pm 0.23$ \\
\hline SDAP & 20 & $76.19 \pm 0.08$ & $78.25 \pm 0.02$ & $69.20 \pm 0.09$ & $55.77 \pm 0.14$ & $54.14 \pm 0.09$ & $42.92 \pm 0.15$ \\
$\alpha$-AP & 20 & $68.32 \pm 0.07$ & $67.94 \pm 0.04$ & $59.47 \pm 0.08$ & $\mathbf{5 8 . 3 1} \pm \mathbf{0 . 1 0}$ & $53.23 \pm 0.21$ & $\mathbf{4 6 . 3 8} \pm \mathbf{0 . 1 2}$ \\
$\omega$-AP & 20 & $68.10 \pm 0.06$ & $67.78 \pm 0.03$ & $59.23 \pm 0.06$ & $58.17 \pm 0.13$ & $53.36 \pm 0.20$ & $46.26 \pm 0.18$ \\
\hline $\mathrm{FP}_{\mu}$ & 38 & $77.14 \pm 0.05$ & $79.41 \pm 0.02$ & $70.42 \pm 0.06$ & $57.35 \pm 0.29$ & $\mathbf{5 6 . 1 6} \pm \mathbf{0 . 1 4}$ & $44.87 \pm 0.34$ \\
$\mathrm{FP}_{a}$ & 38 & $77.19 \pm 0.05$ & $79.46 \pm 0.02$ & $70.47 \pm 0.05$ & $55.53 \pm 0.29$ & $54.81 \pm 0.25$ & $42.63 \pm 0.36$ \\
$\mathrm{FP}_{\mu+a}$ & 76 & $\mathbf{7 7 . 9 4} \pm \mathbf{0 . 0 4}$ & $\mathbf{8 0 . 1 7} \pm \mathbf{0 . 0 3}$ & $\mathbf{7 1 . 4 3} \pm \mathbf{0 . 0 5}$ & $56.62 \pm 0.40$ & $55.68 \pm 0.19$ & $43.90 \pm 0.46$ \\
$\mathrm{TF}_{-A P}$ & 18 & $72.34 \pm 0.05$ & $74.93 \pm 0.04$ & $64.33 \pm 0.06$ & $55.52 \pm 0.09$ & $54.09 \pm 0.07$ & $42.75 \pm 0.09$ \\
\hline
\end{tabular}

TABLE XVI: Classification results per class of the Gray-Potsdam data performed on the partition GrayPostam .

\begin{tabular}{l|c|cccccc}
\hline \hline \multirow{2}{*}{ Method } & \multirow{2}{*}{ Dimension } & \multicolumn{5}{|c}{ Accuracy per class (\%) } \\
\cline { 3 - 8 } & & Background & Trees & Cars & Buildings & Low vegetation & Impervious surfaces \\
\hline Gray-values & 4 & $11.42 \pm 0.70$ & $15.64 \pm 1.46$ & $8.37 \pm 0.26$ & $48.01 \pm 0.89$ & $73.23 \pm 1.48$ & $49.38 \pm 0.94$ \\
AP-maxT & 80 & $33.17 \pm 0.58$ & $23.10 \pm 1.17$ & $47.90 \pm 0.42$ & $62.83 \pm 0.60$ & $\mathbf{7 3 . 8 6} \pm \mathbf{1 . 1 1}$ & $31.50 \pm 0.50$ \\
$\mathrm{AP}-m i n T$ & 80 & $\mathbf{4 7 . 8 3} \pm \mathbf{1 . 0 1}$ & $28.56 \pm 0.68$ & $46.29 \pm 0.29$ & $61.01 \pm 0.35$ & $33.92 \pm 0.47$ & $48.29 \pm 0.88$ \\
$\mathrm{AP}$ & 152 & $45.98 \pm 0.32$ & $45.31 \pm 0.25$ & $77.78 \pm 0.25$ & $80.02 \pm 0.34$ & $37.70 \pm 0.38$ & $46.44 \pm 0.25$ \\
\hline $\mathrm{SDAP}$ & 80 & $46.42 \pm 0.41$ & $41.47 \pm 0.28$ & $74.75 \pm 0.38$ & $77.93 \pm 0.21$ & $37.66 \pm 0.35$ & $46.59 \pm 0.17$ \\
$\alpha$-AP & 80 & $35.68 \pm 1.42$ & $43.50 \pm 0.29$ & $63.38 \pm 0.35$ & $79.05 \pm 0.17$ & $37.86 \pm 0.23$ & $\mathbf{5 9 . 9 1} \pm \mathbf{0 . 3 4}$ \\
$\omega$-AP & 80 & $35.34 \pm 1.29$ & $43.44 \pm 0.26$ & $65.02 \pm 0.42$ & $78.67 \pm 0.15$ & $37.82 \pm 0.43$ & $59.88 \pm 0.36$ \\
\hline $\mathrm{FP}_{\mu}$ & 152 & $46.48 \pm 0.21$ & $\mathbf{4 7 . 4 9} \pm \mathbf{0 . 4 1}$ & $\mathbf{7 9 . 3 0} \pm \mathbf{0 . 4 3}$ & $\mathbf{8 0 . 0 8} \pm \mathbf{0 . 7 3}$ & $36.93 \pm 0.33$ & $46.71 \pm 0.31$ \\
$\mathrm{FP}_{a}$ & 152 & $48.67 \pm 1.36$ & $44.95 \pm 0.30$ & $76.30 \pm 0.38$ & $77.22 \pm 0.67$ & $35.63 \pm 0.30$ & $46.08 \pm 0.33$ \\
$\mathrm{FP}_{\mu+a}$ & 304 & $47.53 \pm 0.62$ & $46.52 \pm 0.36$ & $78.34 \pm 0.44$ & $79.18 \pm 0.99$ & $35.85 \pm 0.41$ & $46.67 \pm 0.47$ \\
$\mathrm{TF}-\mathrm{AP}$ & 72 & $40.13 \pm 0.26$ & $43.30 \pm 0.20$ & $78.68 \pm 0.18$ & $76.15 \pm 0.27$ & $50.54 \pm 0.28$ & $35.73 \pm 0.21$ \\
\hline
\end{tabular}

and $\kappa$ scores, respectively. Comparing the results obtained with those two approaches, we conclude that the results obtained with a single tree are better in general. We attribute this to the fact that training and test pixels share nodes at higher levels of the trees computed on the whole data, leading to more similar attribute values.

In terms of accuracy per class (see Tab. XVIII, we see that, for the classes 'background', 'trees', 'cars' and 'buildings', 


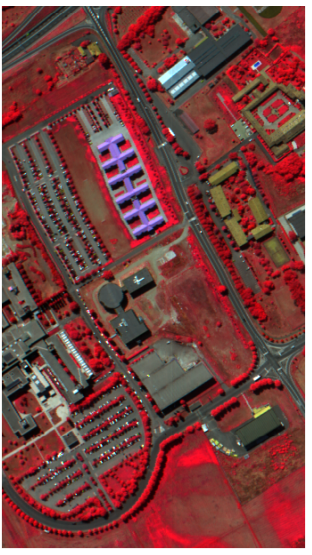

(a) Image

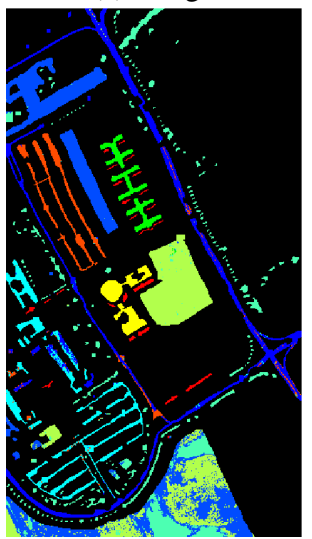

(f) AP-minT

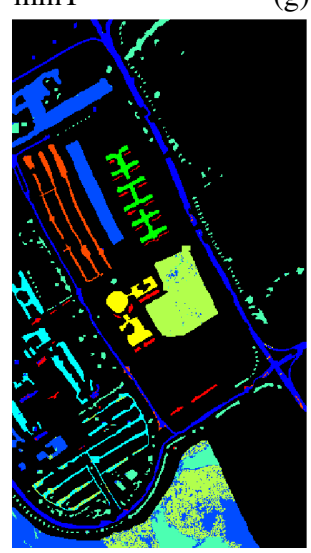

(k) $\mathrm{FP}_{\mu}$

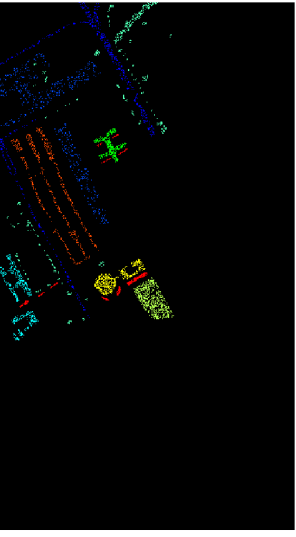

(b) Training set

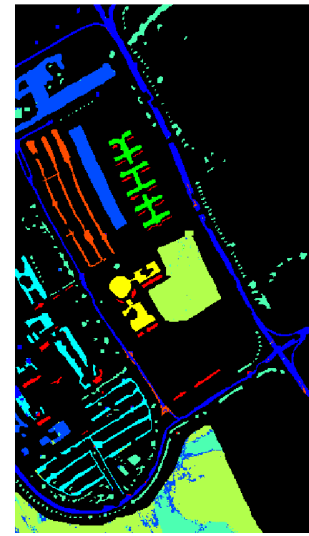

(g) AP

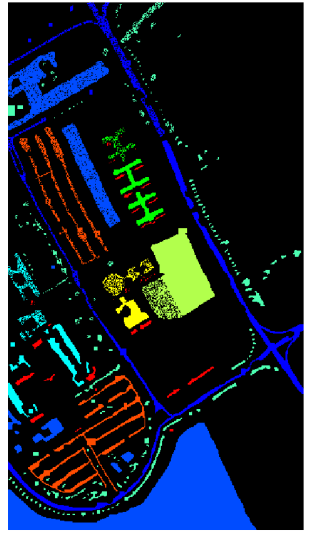

(c) Test set

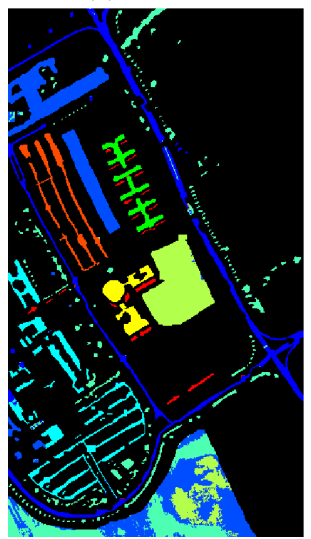

(h) SDAP

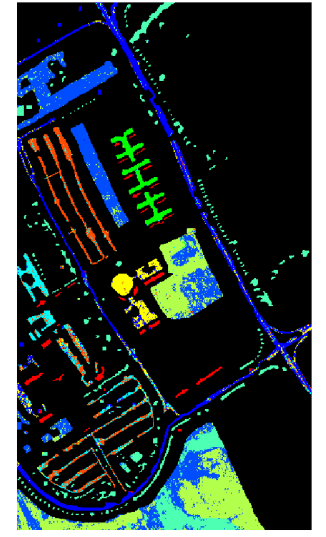

(d) 4 PCs

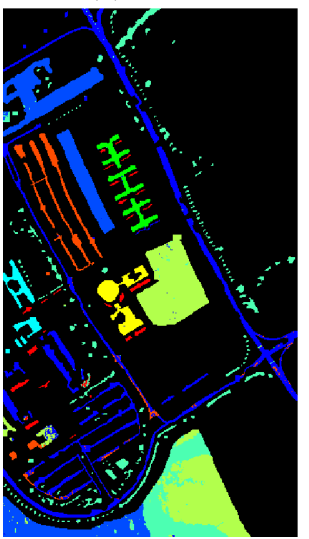

(i) $\alpha-\mathrm{AP}$

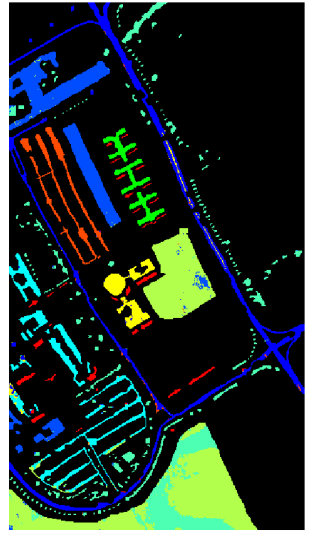

(e) AP-maxT

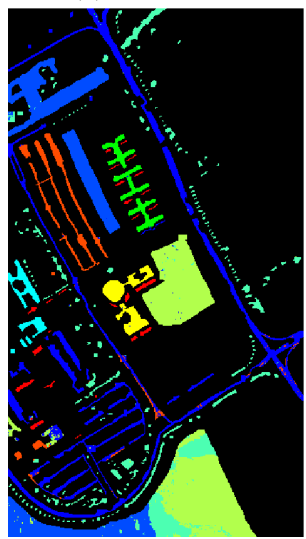

(j) $\omega-\mathrm{AP}$

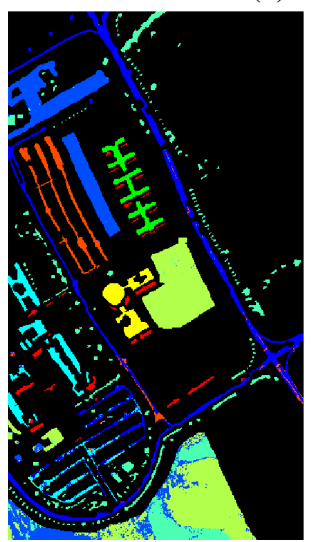

(1) $\mathrm{FP}_{a}$

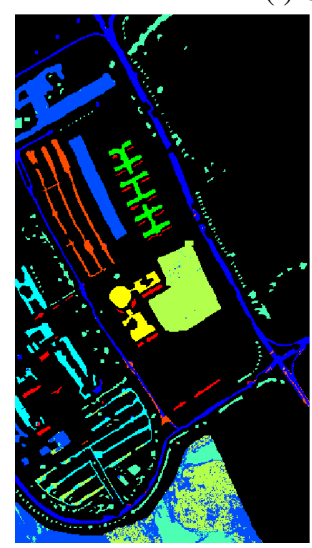

(m) $\mathrm{FP}_{\mu+a}$

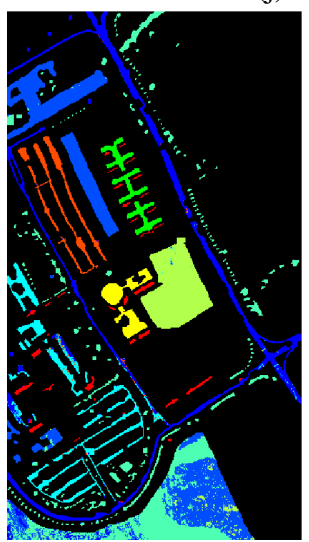

(n) $\mathrm{TF}-\mathrm{AP}$

Fig. 9: Classification results of Pavia dataset (using the partition Pavia $_{2}$ ) based on the proposed training and testing sets. : trees, $\square$ : gravel, $\square$ : meadows, $\square$ : asphalt, $\square$ : metal, $\square$ : bare soil, $\square$ : bitumen, $\square$ : shadows, $\square$ : bricks.

most of the AP-based methods, computed with either one or two independent trees, improve the baseline results. In particular, the largest improvements were observed for the class 'cars', which happens to be composed of the most homogeneous regions in terms of shape and size. Whereas, this was not the case for the 'low vegetation' and 'impervious surface' classes: the performance on those two classes is degraded when APs are used for classification.

From those results, we conclude that the features extracted from APs can be useful in the classification datasets composed of several images extracted from a larger mosaic of images from the same scene. If a single tree representation can be computed from the whole scene, in a semi-supervised scenario, the results can be more promising than if independent trees are computed on each image separately. Moreover, the accuracy scores per class raise the question of whether other geometric and topological attributes could boost the performance for the classes with less homogeneous shapes (like low vegetation and impervious surfaces).

The experiments described in this section highlight some 


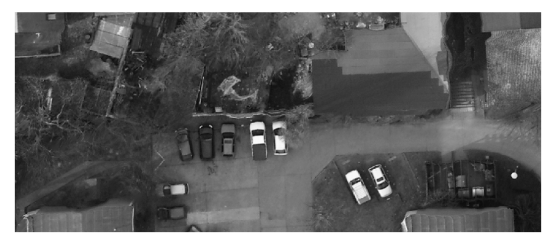

(a) Crop of Gray-Potsdam

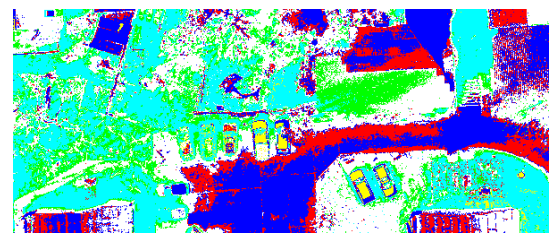

(d) Gray-scale

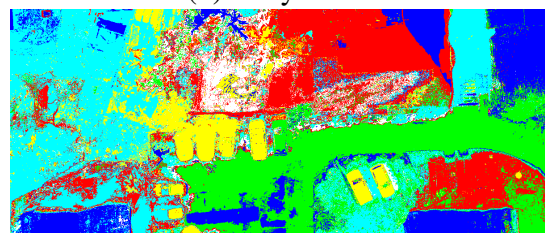

(g) AP

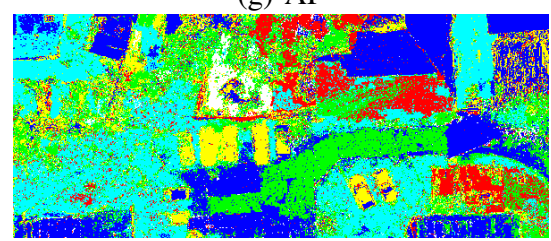

(j) $\omega-\mathrm{AP}$

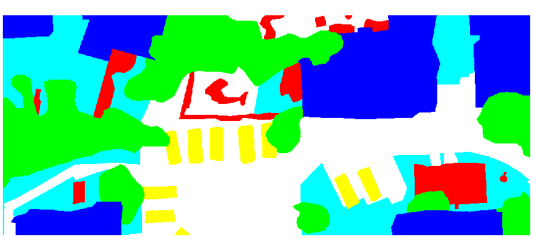

(b) Crop of ground-truth

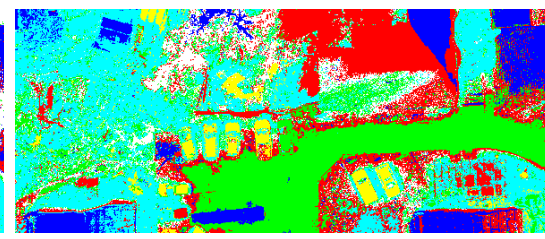

(e) AP-maxT

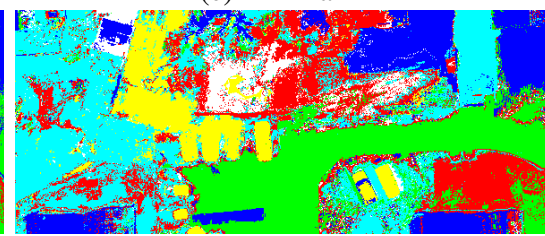

(h) SDAP

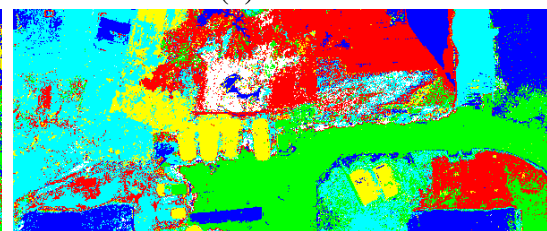

(k) $\mathrm{FP}_{\mu}$

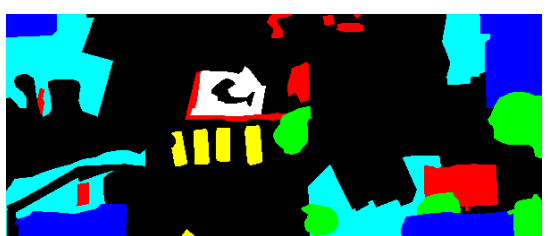

(c) Regions that include training pixels of GrayPotsdam $_{2}$

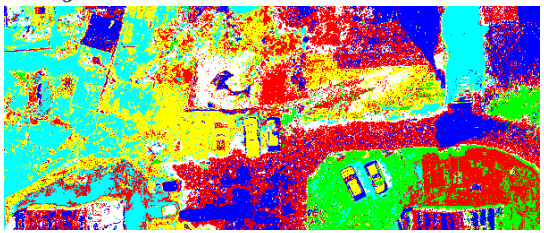

(f) AP-minT

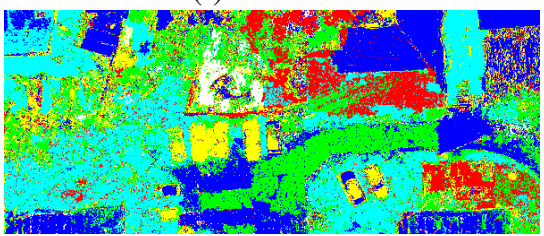

(i) $\alpha-\mathrm{AP}$

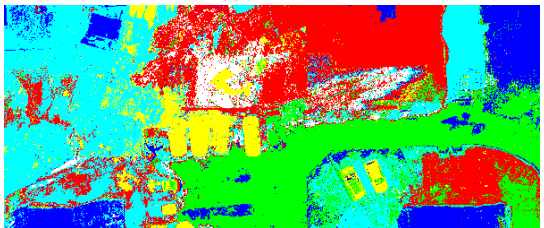

(1) $\mathrm{FP}_{a}$

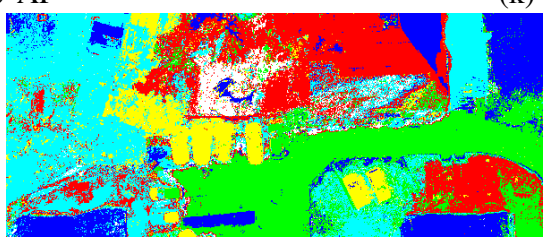

(m) $\mathrm{FP}_{\mu+a}$

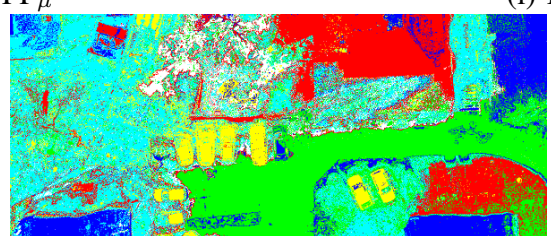

(n) $\mathrm{TF}-\mathrm{AP}$

Fig. 10: Classification results of a crop of Gray-Potsdam corresponding to the results of Table XV $\square$ : impervious surfaces,

$\square$ : buildings, $\square$ : low vegetation, $\square$ : trees, $\square$ : cars, $\square$ : background.

TABLE XVII: Classification results of the Gray-Potsdam dataset with the partition GrayPotsdam 3 following two approaches: training and test features extracted from a single tree computed on the whole data; and from independent trees computed on the training and test images.

\begin{tabular}{l|c|ccc|ccc}
\hline \hline \multirow{2}{*}{ Method } & \multirow{2}{*}{ Dimension } & \multicolumn{2}{|c}{ Classification result (Potsdam 3 , single tree) } & \multicolumn{2}{c}{ Classification result (Potsdam 3 , two trees) } \\
\cline { 3 - 8 } & & OA (\%) & AA (\%) & $\kappa \times 100$ & OA (\%) & AA (\%) & $\kappa \times 100$ \\
\hline Gray-level & 1 & $48.04 \pm 0.67$ & $33.22 \pm 0.14$ & $31.68 \pm 0.58$ & $48.04 \pm 0.67$ & $33.22 \pm 0.14$ & $31.68 \pm 0.58$ \\
AP-maxT & 20 & $50.89 \pm 0.42$ & $39.41 \pm 0.19$ & $35.11 \pm 0.45$ & $41.07 \pm 1.09$ & $33.45 \pm 0.60$ & $25.22 \pm 0.97$ \\
AP-minT & 20 & $36.34 \pm 0.31$ & $32.82 \pm 0.23$ & $21.87 \pm 0.25$ & $37.60 \pm 0.64$ & $31.24 \pm 0.37$ & $22.22 \pm 0.56$ \\
AP & 38 & $47.99 \pm 0.26$ & $40.22 \pm 0.17$ & $32.48 \pm 0.30$ & $48.02 \pm 0.30$ & $37.49 \pm 0.28$ & $31.71 \pm 0.37$ \\
\hline SDAP & 20 & $48.47 \pm 0.31$ & $37.98 \pm 0.28$ & $32.35 \pm 0.39$ & $48.28 \pm 0.32$ & $36.97 \pm 0.38$ & $31.68 \pm 0.37$ \\
$\alpha$-AP & 20 & $53.80 \pm 0.26$ & $43.32 \pm 0.19$ & $40.20 \pm 0.29$ & $46.33 \pm 0.22$ & $31.43 \pm 0.31$ & $26.66 \pm 0.43$ \\
$\omega-A P$ & 20 & $\mathbf{5 3 . 8 9} \pm \mathbf{0 . 2 2}$ & $\mathbf{4 3 . 8 0} \pm \mathbf{0 . 1 8}$ & $\mathbf{4 0 . 3 1} \pm \mathbf{0 . 2 5}$ & $46.21 \pm 0.38$ & $31.05 \pm 0.29$ & $26.00 \pm 0.50$ \\
\hline $\mathrm{FP}_{\mu}$ & 38 & $49.66 \pm 0.22$ & $39.76 \pm 0.17$ & $34.42 \pm 0.27$ & $49.77 \pm 0.64$ & $37.31 \pm 0.26$ & $33.30 \pm 0.67$ \\
$\mathrm{FP}_{a}$ & 38 & $45.03 \pm 0.51$ & $37.99 \pm 0.27$ & $29.61 \pm 0.47$ & $46.58 \pm 0.38$ & $36.33 \pm 0.30$ & $29.57 \pm 0.45$ \\
$\mathrm{FP}_{\mu+a}$ & 76 & $49.82 \pm 0.32$ & $40.70 \pm 0.37$ & $34.89 \pm 0.38$ & $\mathbf{5 1 . 9 2} \pm \mathbf{0 . 3 9}$ & $\mathbf{3 8 . 6 8} \pm \mathbf{0 . 3 3}$ & $\mathbf{3 5 . 6 4} \pm \mathbf{0 . 5 3}$ \\
\hline
\end{tabular}

of the challenges that we can encounter when using APs in different contexts of image classification as, for example, the selection of suitable attributes. Even though the improvements are less significant when compared to the standard data partitions employed in published works (as explained in Section IV), we are still able to benefit from the spatial features extracted from APs. This opens a path for further investigations on the extension of APs for image classification 


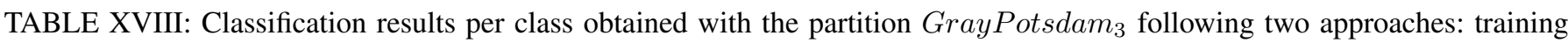
and test features extracted from a single tree computed on the whole data; and from independent trees computed on the training and test images.

\begin{tabular}{|c|c|c|c|c|c|c|c|}
\hline \multirow{2}{*}{ Method } & \multirow{2}{*}{ Trees } & \multicolumn{6}{|c|}{ Accuracy per class (\%) } \\
\hline & & Background & Trees & Cars & Buildings & Low vegetation & Impervious surfaces \\
\hline Gray-level & - & $1.59 \pm 0.94$ & $15.46 \pm 1.25$ & $10.99 \pm 0.40$ & $49.76 \pm 1.90$ & $76.20 \pm 1.05$ & $45.28 \pm 1.35$ \\
\hline \multirow[t]{2}{*}{ AP-maxT } & 1 & $4.14 \pm 0.35$ & $19.17 \pm 0.78$ & $43.18 \pm 0.52$ & $60.55 \pm 0.86$ & $73.20 \pm 0.78$ & $36.20 \pm 0.92$ \\
\hline & 2 & $7.14 \pm 1.16$ & $29.22 \pm 3.02$ & $44.43 \pm 1.09$ & $62.89 \pm 0.93$ & $33.01 \pm 4.28$ & $24.01 \pm 2.68$ \\
\hline \multirow[t]{2}{*}{ AP-minT } & 1 & $29.45 \pm 1.08$ & $38.56 \pm 1.08$ & $25.41 \pm 1.13$ & $40.48 \pm 0.84$ & $42.99 \pm 0.89$ & $20.00 \pm 1.28$ \\
\hline & 2 & $20.89 \pm 0.79$ & $38.66 \pm 2.29$ & $17.87 \pm 0.64$ & $41.74 \pm 1.31$ & $47.67 \pm 1.75$ & $20.58 \pm 2.49$ \\
\hline \multirow[t]{2}{*}{ AP } & 1 & $10.84 \pm 0.26$ & $40.69 \pm 0.74$ & $50.81 \pm 0.71$ & $65.48 \pm 0.63$ & $49.32 \pm 1.40$ & $24.17 \pm 0.77$ \\
\hline & 2 & $5.48 \pm 0.37$ & $38.66 \pm 0.97$ & $38.95 \pm 0.51$ & $67.15 \pm 0.46$ & $48.29 \pm 1.29$ & $26.43 \pm 1.41$ \\
\hline \multirow[t]{2}{*}{ SDAP } & 1 & $8.71 \pm 0.24$ & $34.81 \pm 0.95$ & $40.25 \pm 1.04$ & $67.77 \pm 0.33$ & $52.98 \pm 1.35$ & $23.36 \pm 0.58$ \\
\hline & 2 & $4.98 \pm 0.49$ & $30.96 \pm 0.53$ & $40.03 \pm 1.59$ & $69.60 \pm 0.35$ & $50.58 \pm 0.79$ & $25.64 \pm 0.76$ \\
\hline \multirow[t]{2}{*}{$\alpha-\mathrm{AP}$} & 1 & $9.57 \pm 0.89$ & $34.49 \pm 0.88$ & $43.74 \pm 0.38$ & $68.10 \pm 0.13$ & $54.04 \pm 1.24$ & $49.99 \pm 1.60$ \\
\hline & 2 & $3.94 \pm 0.56$ & $11.46 \pm 2.21$ & $25.43 \pm 1.39$ & $71.73 \pm 0.34$ & $56.21 \pm 1.45$ & $19.84 \pm 0.92$ \\
\hline \multirow[t]{2}{*}{$\omega$-AP } & 1 & $9.43 \pm 0.88$ & $34.39 \pm 0.67$ & $46.55 \pm 0.37$ & $67.97 \pm 0.33$ & $54.37 \pm 0.98$ & $50.07 \pm 1.33$ \\
\hline & 2 & $2.39 \pm 0.68$ & $12.30 \pm 1.90$ & $24.72 \pm 0.74$ & $72.07 \pm 0.87$ & $55.21 \pm 1.76$ & $19.63 \pm 0.78$ \\
\hline \multirow{2}{*}{$\mathrm{FP}_{\mu}$} & 1 & $10.23 \pm 0.24$ & $40.04 \pm 0.84$ & $42.02 \pm 0.62$ & $68.84 \pm 0.45$ & $49.44 \pm 1.06$ & $27.97 \pm 0.90$ \\
\hline & 2 & $4.13 \pm 0.31$ & $36.16 \pm 0.87$ & $35.64 \pm 0.67$ & $71.73 \pm 1.43$ & $52.23 \pm 1.42$ & $23.97 \pm 1.54$ \\
\hline \multirow[t]{2}{*}{$\mathrm{FP}_{a}$} & 1 & $10.27 \pm 0.60$ & $42.05 \pm 1.49$ & $48.75 \pm 1.19$ & $63.71 \pm 1.09$ & $41.17 \pm 1.70$ & $21.99 \pm 1.04$ \\
\hline & 2 & $5.35 \pm 1.10$ & $32.49 \pm 1.20$ & $40.71 \pm 1.33$ & $66.74 \pm 0.93$ & $46.99 \pm 1.45$ & $25.67 \pm 1.66$ \\
\hline \multirow[t]{2}{*}{$\mathrm{FP}_{\mu+a}$} & 1 & $10.0 \pm 0.22$ & $41.32 \pm 1.50$ & $48.38 \pm 1.46$ & $69.90 \pm 0.52$ & $49.26 \pm 1.26$ & $25.32 \pm 0.88$ \\
\hline & 2 & $2.42 \pm 0.39$ & $37.94 \pm 1.18$ & $36.36 \pm 0.81$ & $75.43 \pm 0.43$ & $50.85 \pm 1.69$ & $29.07 \pm 0.83$ \\
\hline
\end{tabular}

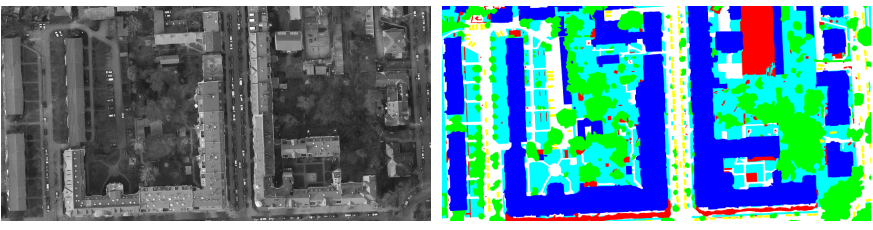

(a)

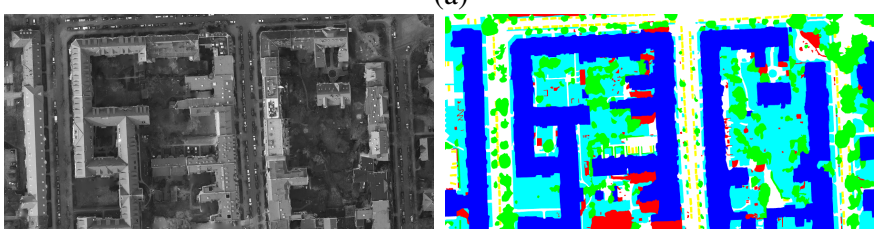

(b)

Fig. 11: Training and testing sets from Gray-Potsdam dataset, made respectively on the upper half (a) and lower half (b). Both grayscale images and their ground-truth are provided.

using multiple images.

\section{FUTURE DIRECTIONS}

Despite a decade of firm advances, and a wide acclaim by the scientific community, many AP related research questions still remain unanswered while new ones are added often due to constant technological advances in terms of image acquisition. The proliferation of satellites and active \& passive sensor types, raises the important question of how to represent and analyze through AP heterogeneous as well as multi-resolution data; e.g. optical and SAR. Likewise, the ever increasing temporal resolutions also present the challenge of handling multi-temporal data in the context of hierarchical image representation and processing. Also, an additional significant research direction is the AP based analysis of multivariate data (either multispectral or hyperspectral), since there is no widely accepted multivariate morphology framework as of yet.
Moreover, given the regular availability of large scale data through missions such as Sentinel-1 and Sentinel-2, more general research directions include the computation of domain invariant features and boosting the already high scalability of AP. Furthermore, given the capacity of AP for effective content description through relatively few training samples, and the ground breaking content description performance of deep networks, the combination of their potentials constitutes a powerful concept worthy of pursuing.

Last but not least, regarding the generalization of APs to real-world scenarios, future directions include experiments with multiple images of a dataset (e.g. training and testing on multiple image patches of the Potsdam dataset) and a study on the generalization capacity of APs across datasets (with similar image resolutions).

\section{CONCLUSION}

APs have replaced morphological profiles as an effective spatial-spectral pixel description tool, and reinforced them in terms of both computational efficiency and flexibility, thus rendering them one of the paramount approaches of their field during the last decade, prior to the advent of deep learning. They have been employed extensively by a plethora of researchers, referenced in hundreds of publications while having been extended in a wide variety of ways.

On the contrary of past works, this survey has provided an extensive review of an entire decade of AP related developments, organized according to each of the AP calculation steps, as well as in terms of adaptation strategies to multivariate data, underlying tree representations, attribute selection and very recent post-processing strategies.

Furthermore, a comprehensive series of experiments has been conducted with multiple datasets, in order to quantify the relative performances of major AP variants using the standard, as well as additional parameters, that have been investigated 
for the first time in the state of the art. And it has turned out that they have a significant effect on classification performance despite being often overlooked: image quantization level and data connectivity. Our results have confirmed that despite their age, the recent AP variants are powerful enough to compete in the case of some datasets even against deep learning. In addition, for the sake of reproducibility, all experiments have been conducted with a publicly accessible software library.

Moreover, one more significant contribution of this survey, is raising the issue of data division in the context of the underlying evaluation protocol. We have underlined the pitfalls of using a single tree structure for the entire image, as is commonly conducted in the state of the art, and proposed a solution through a spatial subdivision of the image with multiple resulting trees to simulate a real deployment scenario. Our findings have shown very important performance gaps that can otherwise lead to false generalization conclusions.

\section{ACKNOWLEDGMENT}

This work was supported by the ANR Multiscale project under the reference ANR-18-CE23-0022. E. Aptoula was supported by the Tubitak project 118E258. The authors would like to thank Prof. Paolo Gamba and ISPRS for making available the Pavia University and Potsdam datasets, respectively.

\section{REFERENCES}

[1] D. A. Landgrebe, Signal Theory Methods in Multispectral Remote Sensing. New York: Wiley, 2003.

[2] M. Pesaresi and J. A. Benediktsson, "A new approach for the morphological segmentation of high-resolution satellite imagery," IEEE Trans. Geosci. Remote Sens., vol. 39, no. 2, pp. 309-320, 2001.

[3] A. Plaza, P. Martinez, R. Perez, and J. Plaza, "A new approach to mixed pixel classification of hyperspectral imagery based on extended morphological profiles," Pattern Recogn., vol. 37, no. 6, pp. 1097-1116, 2004.

[4] J. A. Palmason, J. A. Benediktsson, J. R. Sveinsson, and J. Chanussot, "Classification of hyperspectral data from urban areas using morphological preprocessing and independent component analysis," in Proc. IEEE Int. Geosci. Remote Sens. Symp. (IGARSS), vol. 1, 2005, pp. $176-179$

[5] J. A. Benediktsson, J. A. Palmason, and J. R. Sveinsson, "Classification of hyperspectral data from urban areas based on extended morphological profiles," IEEE Trans. Geosci. Remote Sens., vol. 43, no. 3, pp. 480-491, 2005.

[6] M. Fauvel, J. A. Benediktsson, J. Chanussot, and J. R. Sveinsson, "Spectral and spatial classification of hyperspectral data using svms and morphological profiles," IEEE Trans. Geosci. Remote Sens., vol. 46, no. 11 , pp. 3804-3814, 2008.

[7] M. Dalla Mura, J. A. Benediktsson, B. Waske, and L. Bruzzone, "Morphological attribute profiles for the analysis of very high resolution images," IEEE Trans. Geosci. Remote Sens., vol. 48, no. 10, pp. 3747 3762, 2010.

[8] E. J. Breen, , and R. Jones, "Attribute openings, thinnings, and granulometries," Computer Vision and Image Understanding, vol. 64, no. 3, pp. 377-389, 1996.

[9] L. Najman and M. Couprie, "Building the component tree in quasilinear time," IEEE Transactions on Image Processing, vol. 15, no. 11, pp. 3531-3539, November 2006.

[10] F. Merciol, T. Balem, and S. Lefèvre, "Efficient and large-scale land cover classification using multiscale image analysis," in ESA Conf. on Big Data from Space (BiDS), 2017.

[11] P. Salembier, , A. Oliveras, and L. Garrido, "Antiextensive connected operators for image and sequence processing," IEEE Transactions on Image Processing, vol. 7, no. 4, pp. 555-570, April 1998.

[12] F. Merciol, L. Faucqueur, B. B. Damodaran, P.-Y. Rémy, B. Desclée, F. Dazin, S. Lefèvre, A. Masse, and C. Sannier, "Geobia at the terapixel scale: Toward efficient mapping of small woody features from heterogeneous vhr scenes," ISPRS International Journal of GeoInformation, vol. 8, no. 1, p. 46, 2019.
[13] L. Ma, Y. Liu, X. Zhang, Y. Ye, G. Yin, and B. A. Johnson, "Deep learning in remote sensing applications: A meta-analysis and review," ISPRS journal of photogrammetry and remote sensing, vol. 152, pp. 166-177, 2019.

[14] P. Ghamisi, M. Dalla Mura, and J. A. Benediktsson, "A survey on spectral-spatial classification techniques based on attribute profiles," IEEE Trans. Geosci. Remote Sens., vol. 53, no. 5, pp. 2335-2353, 2015.

[15] P. Ghamisi, E. Maggiori, S. Li, R. Souza, Y. Tarablaka, G. Moser, A. De Giorgi, L. Fang, Y. Chen, M. Chi, et al., "New frontiers in spectral-spatial hyperspectral image classification: The latest advances based on mathematical morphology, markov random fields, segmentation, sparse representation, and deep learning," IEEE geoscience and remote sensing magazine, vol. 6, no. 3, pp. 10-43, 2018.

[16] F. Guiotte, S. Lefèvre, and T. Corpetti, "Rasterization strategies for airborne lidar classification using attribute profiles," in 2019 Joint Urban Remote Sensing Event (JURSE). IEEE, 2019, pp. 1-4.

[17] N. Audebert, B. Le Saux, and S. Lefèvre, "Deep learning for classification of hyperspectral data: a comparative review," IEEE Geosci. Remote Sens. Magaz., vol. 7, no. 2, pp. 159-173, 2019.

[18] M.-T. Pham, S. Lefèvre, E. Aptoula, and L. Bruzzone, "Recent developments from attribute profiles for remote sensing image classification," in ICPRAI, 2018.

[19] M. Dalla Mura, J. Atli Benediktsson, B. Waske, and L. Bruzzone, "Extended profiles with morphological attribute filters for the analysis of hyperspectral data," Int. J. Remote Sens., vol. 31, no. 22, pp. 59755991, 2010.

[20] M. Dalla Mura, A. Villa, J. A. Benediktsson, J. Chanussot, and L. Bruzzone, "Classification of hyperspectral images by using extended morphological attribute profiles and independent component analysis," IEEE Geosci. Remote Sens. Lett., vol. 8, no. 3, pp. 542-546, 2011.

[21] Y. Wu, L. Zheng, D. Xie, R. Zhong, and Q. Chen, "Classification of high-resolution multispectral satellite remote sensing images using extended morphological attribute profiles and independent component analysis," in Ninth International Conference on Digital Image Processing (ICDIP 2017), vol. 10420. International Society for Optics and Photonics, 2017, p. 104203I.

[22] S. Bernabe, P. R. Marpu, A. Plaza, M. Dalla Mura, and J. A. Benediktsson, "Spectral-spatial classification of multispectral images using kernel feature space representation," IEEE Geosci. Remote Sens. Lett., vol. 11, no. 1, pp. 288-292, 2014.

[23] M. Imani and H. Ghassemian, "Attribute profile based feature space discriminant analysis for spectral-spatial classification of hyperspectral images," Computers \& Electrical Engineering, vol. 62, pp. 555-569, 2017.

[24] P. R. Marpu, M. Pedergnana, M. Dalla Mura, S. Peeters, J. A. Benediktsson, and L. Bruzzone, "Classification of hyperspectral data using extended attribute profiles based on supervised and unsupervised feature extraction techniques," Int. J. Image Data Fusion, vol. 3, no. 3, pp. 269-298, 2012.

[25] G. Cavallaro, M. Dalla Mura, J. A. Benediktsson, and L. Bruzzone, "Extended self-dual attribute profiles for the classification of hyperspectral images," IEEE Geosci. Remote Sens. Lett., vol. 12, no. 8, pp. 1690-1694, 2015.

[26] B. B. Damodaran, N. Courty, and S. Lefèvre, "Sparse hilbert schmidt independence criterion and surrogate-kernel-based feature selection for hyperspectral image classification," IEEE Trans. Geosci. Remote Sens., vol. 55, no. 4, pp. 2385-2398, 2017.

[27] E. Aptoula, M. Dalla Mura, and S. Lefèvre, "Vector attribute profiles for hyperspectral image classification," IEEE Trans. Geosci. Remote Sens., vol. 54, no. 6, pp. 3208-3220, 2016.

[28] B. B. Damodaran, J. Höhle, and S. Lefèvre, "Attribute profiles on derived features for urban land cover classification," Photogrammetric Engineering \& Remote Sensing, vol. 83, no. 3, pp. 183-193, 2017.

[29] M.-T. Pham, S. Lefèvre, and F. Merciol, "Attribute profiles on derived textural features for highly textured optical image classification," IEEE Geoscience and Remote Sensing Letters, vol. 15, no. 7, pp. 1125-1129, 2018.

[30] A. Taghipour and H. Ghassemian, "Hyperspectral anomaly detection using attribute profiles," IEEE Geoscience and Remote Sensing Letters, vol. 14, no. 7, pp. 1136-1140, 2017.

[31] G. A. Licciardi, A. Villa, M. Dalla Mura, L. Bruzzone, J. Chanussot, and J. A. Benediktsson, "Retrieval of the height of buildings from worldview-2 multi-angular imagery using attribute filters and geometric invariant moments," IEEE Journal of Selected Topics in Applied Earth Observations and Remote Sensing, vol. 5, no. 1, pp. 71-79, 2012. 
[32] N. Falco, M. Dalla Mura, F. Bovolo, J. A. Benediktsson, and L. Bruzzone, "Change detection in vhr images based on morphological attribute profiles," IEEE Geoscience and Remote Sensing Letters, vol. 10, no. 3, pp. 636-640, 2012.

[33] M. Boldt, A. Thiele, K. Schulz, and S. Hinz, "Sar image segmentation using morphological attribute profiles," The International Archives of Photogrammetry, Remote Sensing and Spatial Information Sciences, vol. 40, no. 3, p. 39, 2014.

[34] L. Xue, X. Yang, and Z. Cao, "Building extraction of sar images using morphological attribute profiles," in Communications, Signal Processing, and Systems. Springer, 2012, pp. 13-21.

[35] P. R. Marpu, K.-S. Chen, C.-Y. Chu, and J. A. Benediktsson, "Spectralspatial classification of polarimetric SAR data using morphological profiles," in Synthetic Aperture Radar (APSAR), 2011 3rd Int. AsiaPacific Conf., 2011, pp. 1-3.

[36] A. Tombak, E. Aptoula, and K. Kayabol, "Pixel-based classification of sar images using features," in Proceedings of 26th Signal Processing and Communications Applications Conference, Cesme, Turkey, 2018.

[37] M. Boldt, K. Schulz, A. Thiele, and S. Hinz, "Using morphological differential attribute profiles for change categorization in high resolution sar images," Inter. Arch. Photogramm. Remote Sens. Spatial Inf. Sci, vol. 1, no. 1, pp. 29-34, 2013.

[38] M. Boldt, A. Thiele, K. Schulz, and S. Hinz, "Feature extraction for change analysis in sar time series," in Earth Resources and Environmental Remote Sensing/GIS Applications VI, vol. 9644. International Society for Optics and Photonics, 2015, p. 964410.

[39] M. Boldt, A. Thiele, K. Schulz, F. J. Meyer, and S. Hinz, "Practical approach for synthetic aperture radar change analysis in urban environments," Journal of Applied Remote Sensing, vol. 13, no. 3, p. 034528, 2019.

[40] S. Lefèvre and E. Aptoula, "Morphological tools for spatial and multiscale analysis of passive microwave remote sensing data," in 2016 14th Specialist Meeting on Microwave Radiometry and Remote Sensing of the Environment (MicroRad). IEEE, 2016, pp. 145-150.

[41] D. Mongus, N. Lukač, D. Obrul, and B. Žalik, "Detection of planar points for building extraction from lidar data based on differential morphological and attribute profiles," in VCM 2013-The ISPRS Workshop on $3 D$ Virtual City Modeling, vol. 2. Univerza v Mariboru, Fakulteta za elektrotehniko, računalništvo in informatiko, 2013, pp. 21-26.

[42] M. Pedergnana, P. R. Marpu, M. Dalla Mura, J. A. Benediktsson, and L. Bruzzone, "Classification of remote sensing optical and lidar data using extended attribute profiles," IEEE J. Sel. Topics Sig. Proc., vol. 6 , no. 7, pp. 856-865, 2012.

[43] W. Liao, J. Chanussot, M. Dalla Mura, X. Huang, R. Bellens, S. Gautama, and W. Philips, "Taking optimal advantage of fine spatial resolution: Promoting partial image reconstruction for the morphological analysis of very-high-resolution images," IEEE Geosci. Remote Sens. Magaz., vol. 5, no. 2, pp. 8-28, 2017

[44] F. Guiotte, S. Lefèvre, and T. Corpetti, "Voxel-based attribute profiles on lidar data for land cover mapping," in IGARSS 2019-2019 IEEE International Geoscience and Remote Sensing Symposium. IEEE, 2019, pp. 2491-2494.

[45] C. Tuna, F. Merciol, and S. Lefèvre, "Attribute profiles for satellite image time series," in IGARSS 2019-2019 IEEE International Geoscience and Remote Sensing Symposium. IEEE, 2019, pp. 126-129.

[46] C. Tuna, B. Mirmahboub, F. Merciol, and S. Lefèvre, "Component trees for image sequences and streams," Pattern Recognition Letters, vol. 129, pp. 255-262, 2020.

[47] M. Zhang, P. Ghamisi, and W. Li, "Classification of hyperspectral and lidar data using extinction profiles with feature fusion," Remote Sensing Letters, vol. 8, no. 10, pp. 957-966, 2017.

[48] C. Kwan, D. Gribben, B. Ayhan, S. Bernabe, A. Plaza, and M. Selva, "Improving land cover classification using extended multi-attribute profiles (emap) enhanced color, near infrared, and lidar data," Remote Sensing, vol. 12, no. 9, p. 1392, 2020.

[49] P. Ghamisi, R. Souza, J. A. Benediktsson, L. Rittner, R. Lotufo, and X. X. Zhu, "Hyperspectral data classification using extended extinction profiles," IEEE Geoscience and Remote Sensing Letters, vol. 13, no. 11, pp. 1641-1645, 2016.

[50] D. Hong, L. Gao, R. Hang, B. Zhang, and J. Chanussot, "Deep encoderdecoder networks for classification of hyperspectral and lidar data," IEEE Geoscience and Remote Sensing Letters, 2020.

[51] P. Bosilj, E. Kijak, and S. Lefèvre, "Partition and inclusion hierarchies of images: A comprehensive survey," Journal of Imaging, vol. 4, no. 2, p. 33,2018 .
[52] C. Ronse, "Ordering partial partitions for image segmentation and filtering: merging, creating and inflating blocks," Journal of Mathematical Imaging and Vision, vol. 49, no. 2, pp. 202-233, 2014.

[53] M. Dalla Mura, J. Benediktsson, and L. Bruzzone, "Self-dual attribute profiles for the analysis of remote sensing images," in Int. Symp. Math. Morpho. Appl. Sig. Image Proc., 2011, pp. 320-330.

[54] P. Monasse and F. Guichard, "Scale-space from a level lines tree," Journal of Visual Communication and Image Representation, vol. 11, no. 2, pp. 224-236, 2000 .

[55] G. Cavallaro, M. Dalla Mura, J. A. Benediktsson, and A. Plaza, "Remote sensing image classification using attribute filters defined over the tree of shapes," IEEE Trans. Geosci. Remote Sens., vol. 54, no. 7, pp. 3899-3911, 2016.

[56] P. Bosilj, B. B. Damodaran, E. Aptoula, M. Dalla Mura, and S. Lefèvre, "Attribute profiles from partitioning trees," in Int. Symp. Math. Morpho. Its Appl. Sig. Image Proc., 2017, pp. 381-392.

[57] P. Soille, "Constrained connectivity for hierarchical image partitioning and simplification," IEEE Trans. Patt. Anal. Mach. Intell., vol. 30, no. 7, pp. 1132-1145, 2008.

[58] P. Soille, "On genuine connectivity relations based on logical predicates," in International Conference on Image Analysis and Processing, 2007, pp. 487-492.

[59] S. Lefèvre, L. Chapel, and F. Merciol, "Hyperspectral image classification from multiscale description with constrained connectivity and metric learning," in IEEE Wksh. Hyper. Image and Sig. Proc.: Evol. Remote Sens. (WHISPERS), 2014, pp. 1-4.

[60] S. G. Koç, E. Aptoula, P. Bosilj, B. B. Damodaran, M. Dalla Mura, and S. Lefevre, "A comparative noise robustness study of tree representations for attribute profile construction," in 2017 25th Signal Processing and Communications Applications Conference (SIU). IEEE, 2017, pp. $1-4$.

[61] P. Ghamisi, J. A. Benediktsson, and J. R. Sveinsson, "Automatic spectral-spatial classification framework based on attribute profiles and supervised feature extraction," IEEE Trans. Geosci. Remote Sens., vol. 52, no. 9, pp. 5771-5782, 2014.

[62] P. Ghamisi, J. A. Benediktsson, G. Cavallaro, and A. Plaza, "Automatic framework for spectral-spatial classification based on supervised feature extraction and morphological attribute profiles," IEEE Journal of Selected Topics in Applied Earth Observations and Remote Sensing, vol. 7, no. 6, pp. 2147-2160, 2014.

[63] P. R. Marpu, M. Pedergnana, M. Dalla Mura, J. A. Benediktsson, and L. Bruzzone, "Automatic generation of standard deviation attribute profiles for spectral-spatial classification of remote sensing data," IEEE Geosci. Remote Sens. Lett., vol. 10, no. 2, pp. 293-297, 2013.

[64] A. Das, K. Bhardwaj, S. Patra, and L. Bruzzone, "A novel threshold detection technique for the automatic construction of attribute profiles in hyperspectral images," IEEE Journal of Selected Topics in Applied Earth Observations and Remote Sensing, vol. 13, pp. 1374-1384, 2020.

[65] A. Das, K. Bhardwaj, and S. Patra, "Morphological complexity profile for the analysis of hyperspectral images," in 2018 4th International Conference on Recent Advances in Information Technology (RAIT). IEEE, 2018, pp. 1-6.

[66] K. Bhardwaj, S. Patra, and L. Bruzzone, "Threshold-free attribute profile for classification of hyperspectral images," IEEE Transactions on Geoscience and Remote Sensing, vol. 57, no. 10, pp. 7731-7742, 2019.

[67] U. Bhangale, S. S. Durbha, R. L. King, N. H. Younan, and R. Vatsavai, "High performance gpu computing based approaches for oil spill detection from multi-temporal remote sensing data," Remote Sensing of Environment, vol. 202, pp. 28-44, 2017.

[68] E. Aptoula, "Hyperspectral image classification with multidimensional attribute profiles," IEEE Geosci. Remote Sens. Lett., vol. 12, no. 10, pp. 2031-2035, 2015.

[69] Z. Mahmood, G. Thoonen, and P. Scheunders, "Automatic threshold selection for morphological attribute profiles," in Proc. IEEE Int. Geosci. Remote Sens. Symp. (IGARSS), 2012, pp. 4946-4949.

[70] M. Pedergnana, P. R. Marpu, M. Dalla Mura, J. A. Benediktsson, and L. Bruzzone, "A novel technique for optimal feature selection in attribute profiles based on genetic algorithms," IEEE Trans. Geosci. Remote Sens., vol. 51, no. 6, pp. 3514-3528, 2013.

[71] G. Cavallaro, N. Falco, M. Dalla Mura, L. Bruzzone, and J. A. Benediktsson, "Automatic threshold selection for profiles of attribute filters based on granulometric characteristic functions," in Int. Symp. Math. Morpho. Its Appl. Sig. Image Proc., 2015, pp. 169-181.

[72] G. Cavallaro, N. Falco, M. Dalla Mura, and J. A. Benediktsson, "Automatic attribute profiles," IEEE Trans. Image Processing, vol. 26, no. 4, pp. 1859-1872, 2017. 
[73] K. Bhardwaj and S. Patra, "An unsupervised technique for optimal feature selection in attribute profiles for spectral-spatial classification of hyperspectral images," ISPRS Journal of Photogrammetry and Remote Sensing, vol. 138, pp. 139-150, 2018.

[74] E. Aptoula and S. Guner Koc, "Attribute profiles without thresholds," in Proc. IEEE Int. Geosci. Remote Sens. Symp. (IGARSS), 2018.

[75] U. Derbashi and E. Aptoula, "Attribute profiles without thresholds through histogram based tree path description," in Proc. IEEE Mediterranean and Middle-East Geosci. and Remote Sens. Symp. (M2GRSS), 2020 .

[76] P. Salembier and M. H. F. Wilkinson, "Connected operators," IEEE Signal Processing Magazine, pp. 136-157, November 2009.

[77] E. R. Urbach, J. B. T. M. Roerdink, and M. H. F. Wilkinson, "Connected shape-size pattern spectra for rotation and scale-invariant classification of gray-scale images," IEEE Trans. Patt. Anal. Mach. Intell., vol. 29, no. 2, pp. 272-285, February 2007.

[78] G. Cavallaro, M. Dalla Mura, J. A. Benediktsson, and L. Bruzzone, "A comparison of self-dual attribute profiles based on different filter rules for classification," in Proc. IEEE Int. Geosci. Remote Sens. Symp. (IGARSS), 2014, pp. 1265-1268.

[79] B. Demir and L. Bruzzone, "Histogram-based attribute profiles for classification of very high resolution remote sensing images," IEEE Trans. Geosci. Remote Sens., vol. 54, no. 4, pp. 2096-2107, 2016.

[80] M.-T. Pham, S. Lefèvre, and E. Aptoula, "Local feature-based attribute profiles for optical remote sensing image classification," IEEE Trans. Geosci. Remote Sens., vol. 56, no. 2, pp. 1199-1212, 2018.

[81] R. Battiti, B. Demir, and L. Bruzzone, "Compressed histogram attribute profiles for the classification of VHR remote sensing images," in SPIE Remote Sens., 2015, pp. 96430R-96430R.

[82] M.-T. Pham, S. Lefèvre, E. Aptoula, and B. B. Damodaran, "Classification of VHR remote sensing images using local feature-based attribute profiles," in Proc. IEEE Int. Geosci. Remote Sens. Symp. (IGARSS), 2017, pp. 1083-1086.

[83] B. Song, J. Li, M. Dalla Mura, P. Li, A. Plaza, J. M. Bioucas-Dias, J. A Benediktsson, and J. Chanussot, "Remotely sensed image classification using sparse representations of morphological attribute profiles," IEEE Trans. Geosci. Remote Sens., vol. 52, no. 8, pp. 5122-5136, 2014

[84] J. Xia, M. Dalla Mura, J. Chanussot, P. Du, and X. He, "Random subspace ensembles for hyperspectral image classification with extended morphological attribute profiles," IEEE Trans. Geosci. Remote Sens., vol. 53, no. 9, pp. 4768-4786, 2015.

[85] R. Bao, J. Xia, M. Dalla Mura, P. Du, J. Chanussot, and J. Ren, "Combining morphological attribute profiles via an ensemble method for hyperspectral image classification," IEEE Geosci. Remote Sens. Lett., vol. 13, no. 3, pp. 359-363, 2016.

[86] E. Aptoula, M. C. Ozdemir, and B. Yanikoglu, "Deep learning with attribute profiles for hyperspectral image classification," IEEE Geosci. Remote Sens. Lett., vol. 13, no. 12, pp. 1970-1974, 2016.

[87] T. Tian, L. Gao, W. Song, K.-K. R. Choo, and J. He, "Feature extraction and classification of VHR images with attribute profiles and convolutional neural networks," Multimedia Tools and Applications, vol. 77, no. 14, pp. 18637-18656, 2018.

[88] H. Teffahi, H. Yao, S. Chaib, and N. Belabid, "A novel spectral-spatial classification technique for multispectral images using extended multi-attribute profiles and sparse autoencoder," Remote Sensing Letters, vol. 10, no. 1, pp. 30-38, 2019. [Online]. Available: https://doi.org/10.1080/2150704X.2018.1523581

[89] Ş. G. Koç and E. Aptoula, "Hyperspectral image classification with convolutional networks trained with self-dual attribute profiles," in 2017 25th Signal Processing and Communications Applications Conference (SIU). IEEE, 2017, pp. 1-4.

[90] K. Zhu, Y. Chen, P. Ghamisi, X. Jia, and J. A. Benediktsson, "Deep convolutional capsule network for hyperspectral image spectral and spectral-spatial classification," Remote Sensing, vol. 11, no. 3, p. 223, 2019.

[91] P. Ghamisi, R. Souza, J. A. Benediktsson, X. X. Zhu, L. Rittner and R. A. Lotufo, "Extinction profiles for the classification of remote sensing data," IEEE Trans. Geosci. Remote Sens., vol. 54, no. 10, pp. 5631-5645, 2016.

[92] J. Xia, P. Ghamisi, N. Yokoya, and A. Iwasaki, "Random forest ensembles and extended multiextinction profiles for hyperspectral image classification," IEEE Transactions on Geoscience and Remote Sensing, vol. 56, no. 1, pp. 202-216, 2018.

[93] L. Fang, N. He, S. Li, P. Ghamisi, and J. A. Benediktsson, "Extinction profiles fusion for hyperspectral images classification," IEEE Transactions on Geoscience and Remote Sensing, vol. 56, no. 3, pp. 1803-1815, 2017.
[94] W. Liao, M. Dalla Mura, J. Chanussot, R. Bellens, and W. Philips, "Morphological attribute profiles with partial reconstruction," IEEE Trans. Geosci. Remote Sens., vol. 54, no. 3, pp. 1738-1756, 2016.

[95] D. Hong, X. Wu, P. Ghamisi, J. Chanussot, N. Yokoya, and X. X. Zhu, "Invariant attribute profiles: A spatial-frequency joint feature extractor for hyperspectral image classification," IEEE Transactions on Geoscience and Remote Sensing, 2020.

[96] M.-T. Pham, E. Aptoula, and S. Lefèvre, "Feature profiles from attribute filtering for remote sensing image classification," IEEE J. Sel. Topics Appl. Earth Observations Remote Sens., vol. 11, no. 1, pp. 249256, 2018.

[97] M.-T. Pham, E. Aptoula, and S. Lefèvre, "Classification of remote sensing images using attribute profiles and feature profiles from different trees: a comparative study," in IGARSS 2018-2018 IEEE International Geoscience and Remote Sensing Symposium. IEEE, 2018, pp. 45114514.

[98] B. Perret, G. Chierchia, J. Cousty, S. Guimarães, Y. Kenmochi, and L. Najman, "Higra: Hierarchical graph analysis," SoftwareX, vol. 10, p. $100335,2019$.

[99] D. Pascale, "A review of rgb color spaces," Tech. Rep., 2003. [Online]. Available: http://www.babelcolor.com/index_htm_files/A\% 20review\%20of\%20RGB\%20color\%20spaces.pdf

[100] A. Liaw, M. Wiener, et al., "Classification and regression by randomForest," $R$ news, vol. 2, no. 3, pp. 18-22, 2002.

[101] P. Ghamisi, B. Höfle, and X. X. Zhu, "Hyperspectral and lidar data fusion using extinction profiles and deep convolutional neural network," IEEE Journal of Selected Topics in Applied Earth Observations and Remote Sensing, vol. 10, no. 6, pp. 3011-3024, 2016.

[102] P. G. Bascoy, P. Quesada-Barriuso, D. B. Heras, F. Argüello, B. Demir and L. Bruzzone, "Extended attribute profiles on gpu applied to hyperspectral image classification," The Journal of Supercomputing, vol. 75, no. 3, pp. 1565-1579, 2019.

[103] Y. Li, H. Zhang, and Q. Shen, "Spectral-spatial classification of hyperspectral imagery with $3 \mathrm{~d}$ convolutional neural network," Remote Sensing, vol. 9, no. 1, p. 67, 2017.

[104] M. H. Wilkinson, "Hyperconnectivity, attribute-space connectivity and path openings: Theoretical relationships," in International Symposium on Mathematical Morphology and Its Applications to Signal and Image Processing. Springer, 2009, pp. 47-58.

[105] G. K. Ouzounis and M. H. Wilkinson, "Mask-based second-generation connectivity and attribute filters," IEEE Transactions on Pattern Analysis and Machine Intelligence, vol. 29, no. 6, pp. 990-1004, 2007. 